\title{
Local stationarity and time-inhomogeneous Markov chains
}

\author{
Lionel Truquet ${ }^{* i}$
}

\begin{abstract}
In this paper, we study a notion of local stationarity for discrete time Markov chains which is useful for applications in statistics. In the spirit of some locally stationary processes introduced in the literature, we consider triangular arrays of time-inhomogeneous Markov chains, defined by some families of contracting Markov kernels. Using the Dobrushin's contraction coefficients for various metrics, we show that the distribution of such Markov chains can be approximated locally with the distribution of ergodic Markov chains and we also study some mixing properties. From our approximation results in Wasserstein metrics, we recover several properties obtained for autoregressive processes. Moreover, using the total variation distance or more generally some distances induced by a drift function, we consider new models, such as finite state space Markov chains with time-varying transition matrices or some timevarying versions of integer-valued autoregressive processes. For these two examples, nonparametric kernel estimation of the transition matrix is discussed.
\end{abstract}

\section{Introduction}

Time-inhomogeneous Markov chains have received much less attention in the literature than the homogeneous case. Such chains have been studied mainly for their long-time behavior, often in connexion with the convergence of stochastic algorithms. An introduction to inhomogeneous Markov chains and their use in Monte Carlo methods can be found in Winkler (1995). More recent quantitative results for their long time behavior can be found for instance in Douc et al. (2004), Saloff-Coste and Zúñiga (2007), or Saloff-Coste and Zúñiga (2011). In this paper, we consider convergence properties of nonhomogeneous Markov chains but with a different perspective, motivated by applications in mathematical statistics and in the spirit of the notion of local stationarity introduced by Dahlhaus (1997). Locally stationary processes have received a considerable attention over the last twenty years, in particular for their ability to model data sets for which time-homogeneity is unrealistic. Locally stationary autoregressive processes (here with one lag for simplicity) can be defined by modifying a recursive equation followed by a stationary process. If

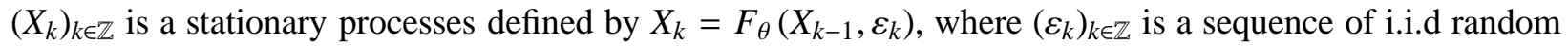
variables and $\theta \in \Theta$ is a parameter, its locally stationary version is usually defined recursively by

$$
X_{n, k}=F_{\theta(k / n)}\left(X_{n, k-1}, \varepsilon_{k}\right), \quad 1 \leq k \leq n,
$$

where $\theta:[0,1] \rightarrow \Theta$ is a smooth function. This formalism was exploited for defining locally stationary versions of classical time-homogeneous autoregressive processes. See for instance Dahlhaus and Subba Rao (2006),

${ }^{*}$ UMR 6625 CNRS IRMAR, University of Rennes 1, Campus de Beaulieu, F-35042 Rennes Cedex, France and

'Campus de Ker-Lann, rue Blaise Pascal, BP 37203, 35172 Bruz cedex, France. Email: lionel.truquet@ensai.fr. 
Subba Rao (2006) or Vogt (2012). The term local stationarity comes from the fact that, under some regularity conditions, if $k / n$ is close to a point $u$ of $[0,1], X_{n, k}$ is close in some sense to $X_{k}(u)$ where $\left(X_{k}(u)\right)_{k \in \mathbb{Z}}$ is the stationary process defined by

$$
X_{k}(u)=F_{\theta(u)}\left(X_{k-1}(u), \varepsilon_{k}\right), \quad k \in \mathbb{Z} .
$$

Though local stationary processes defined recursively are examples of time-inhomogeneous Markov chains, the properties of these processes are usually derived using this particular autoregressive representation and without exploiting the link with Markov chains. This is one the main difference with respect to stationary processes for which the connection between autoregressive processes and Markov chains has been widely used. See for example the classical textbook of Meyn and Tweedie (2009) for many examples of iterative systems studied using Markov chains properties. As a limitation, the simple case of a locally stationary version of finite state space Markov chains has not been considered in the literature.

In this paper, we consider general Markov chains models which will generalize the existing (Markovian) locally stationary processes. Since we do not work directly with autoregressive representations, our definition of local stationarity is based on the approximation of the finite dimensional distributions of the chain with that of some ergodic Markov chains. Let us now give the framework used in the rest of the paper. Let $(E, d)$ be a metric space, $\mathcal{B}(E)$ its corresponding Borel $\sigma$-field and $\left\{Q_{u}: u \in[0,1]\right\}$ a family of Markov kernels on $(E, \mathcal{B}(E))$. By convention, we set $Q_{u}=Q_{0}$ when $u<0$. We will consider triangular arrays $\left\{X_{n, j}: j \leq n, n \in \mathbb{Z}^{+}\right\}$such that for all $n \in \mathbb{Z}^{+}$, the sequence $\left(X_{n, j}\right)_{j \leq n}$ is a non homogeneous Markov chain such that

$$
\left.\mathbb{P}\left(X_{n, k} \in A \mid X_{n, k-1}=x\right)\right)=Q_{k / n}(x, A), \quad k \leq n .
$$

In the sequel the family $\left\{Q_{u}: u \in[0,1]\right\}$ of Markov kernels will always satisfy some regularity conditions and contraction properties. Precise assumptions will be given in three following sections, but from now on, we assume here that for all $u \in[0,1], Q_{u}$ has a single invariant probability denoted by $\pi_{u}$. For all positive integer $j$ and all integer $k$ such that $k+j-1 \leq n$, we denote by $\pi_{k, j}^{(n)}$ the probability distribution of the vector $\left(X_{n, k}, X_{n, k+1}, \ldots, X_{n, k+j-1}\right)$ and by $\pi_{u, j}$ the corresponding finite dimensional distribution for the ergodic chain with Markov kernels $Q_{u}$. Loosely speaking, the triangular array will be said locally stationary if for all positive integer $j$, the probability distribution $\pi_{k, j}^{(n)}$ is close to $\pi_{u, j}$ when the ratio $k / n$ is close to $u$. A formal definition is given below. For an integer $j \geq 1$, we denote by $\mathcal{P}\left(E^{j}\right)$ the set of probability measures on $E^{j}$.

Definition 1. The triangular array of non-homogeneous Markov chains $\left\{X_{n, k}, n \in \mathbb{Z}^{+}, k \leq n\right\}$ is said to be locally stationary if for all integer $j \geq 1$, there exists a metric $\vartheta_{j}$ on $\mathcal{P}\left(E^{j}\right)$, metrizing the topology of weak convergence, such that the two following conditions are satisfied.

1. The application $u \mapsto \pi_{u, j}$ is continuous.

2. $\lim _{n \rightarrow \infty} \sup _{k \leq n-j+1} \vartheta_{j}\left(\pi_{k, j}^{(n)}, \pi_{\frac{k}{n}, j}\right)=0$

In particular, under the two conditions of Definition 1, for all continuous and bounded function $f: E^{j} \rightarrow$ $\mathbb{R}$ and some integers $k=k_{n} \leq n-j+1$ such that $\lim _{n \rightarrow \infty} k / n=u \in[0,1]$, we have

$$
\lim _{n \rightarrow \infty} \mathbb{E} f\left(X_{n, k}, \ldots, X_{n, k+j-1}\right)=\lim _{n \rightarrow \infty} f d \pi_{k, j}^{(n)}=\mathbb{E} f\left(X_{1}(u), \ldots, X_{j}(u)\right)=\int f d \pi_{u, j},
$$

where $\left(X_{k}(u)\right)_{k \in \mathbb{Z}}$ denotes a stationary Markov chain with transition $Q_{u}$. In this paper, Condition 1 will always hold from the Hölder continuity properties that we will assume for the application $u \mapsto Q_{u}$. Of 
course, the metrics $\vartheta_{j}$ will of the same nature for different integers $j$, e.g the total variation distance on $\mathcal{P}\left(E^{j}\right)$.

In this paper, we will consider three type of metrics on $\mathcal{P}(E)$ for approximating $\pi_{k}^{(n)}$ by $\pi_{k / n}$ or $\pi_{u}$ (and in a second step for approximating an arbitrary finite dimensional distribution) and deriving mixing properties of these triangular arrays. We will extensively make use of the so-called Dobrushin's contraction coefficient. In Section 2, we consider the total variation distance. This is the metric for which the contraction coefficient for Markov kernels has been originally introduced by Dobrushin (1956). Contraction properties of the kernels $Q_{u}$ or their iteration with respect to this metric will enable us to consider a model of nonhomogeneous finite state space Markov chains for which we will study a nonparametric estimator of the time-varying transition matrix. In Section 3, we consider contraction properties for Wasserstein metrics. The contraction coefficient for the Wasserstein metric of order 1 has been first considered by Dobrushin (1970) for giving sufficient conditions under which a system of conditional distributions defines a unique joint distribution. We will consider more generally the Wasserstein metric of order $p \geq 1$. This type of metric is very well adapted for recovering some results obtained for autoregressive processes with time-varying coefficients. Finally, in Section 4, we consider Markov kernels satisfying drift and minoration conditions ensuring geometric ergodicity and for which Hairer and Mattingly (2011) have recently found a contraction property for a metric induced by a modified drift function. We illustrate this third approach with the statistical inference of some integer-valued autoregressive processes with time-varying coefficients.

\section{Total variation distance and finite state space Markov chains}

Let us first give some notations that we will extensively use in the sequel. If $\mu \in \mathcal{P}(E)$ and $R$ is a probability kernel from $(E, \mathcal{B}(E))$ to $(E, \mathcal{B}(E))$, we will denote by $\mu R$ the probability measure defined by

$$
\mu R(A)=\int R(x, A) d \mu(x), \quad A \in \mathcal{B}(E) .
$$

Moreover if $f: E \rightarrow \mathbb{R}$ is a measurable function, we set $\mu f=\int f d \mu$ and $R f: E \rightarrow \mathbb{R}$ will be the function defined by $R f(x)=\int R(x, d y) f(y), x \in E$, provided these integrals are well defined. Finally, the Dirac measure at point $x \in E$ is denoted by $\delta_{x}$.

\subsection{Contraction and approximation result for the total variation distance}

The total variation distance between two probability measures $\mu, v \in \mathcal{P}(E)$ is defined by

$$
\|\mu-v\|_{T V}=\sup _{A \in \mathcal{B}(E)}|\mu(A)-v(A)|=\frac{1}{2} \sup _{\|f\|_{\infty} \leq 1}\left|\int f d \mu-\int f d v\right|,
$$

where for a measurable function $f: E \rightarrow \mathbb{R},\|f\|_{\infty}=\sup _{x \in E}|f(x)|$.

For the family $\left\{Q_{u}: u \in[0,1]\right\}$, the following assumptions will be needed.

A1 There exist an integer $m \geq 1$ and $r \in(0,1)$ such that for all $(u, x, y) \in[0,1] \times E^{2}$,

$$
\left\|\delta_{x} Q_{u}^{m}-\delta_{y} Q_{u}^{m}\right\|_{T V} \leq r .
$$

A2 There exist a positive real number $L$ and $\kappa \in(0,1)$ such that for all $(u, v, x) \in[0,1]^{2} \times E$,

$$
\left\|\delta_{x} Q_{u}-\delta_{x} Q_{v}\right\|_{T V} \leq L|u-v|^{\kappa} .
$$


The Dobrushin contraction coefficient of Markov kernel $R$ on $(E, \mathcal{B}(E))$ is defined by $c(R)=\sup _{(x, y) \in E^{2}}\left\|\delta_{x} R-\delta_{y} R\right\|_{T V}$. We have $c(R) \in[0,1]$. Hence, assumption $\mathbf{A 1}$ means that $\sup _{u \in[0,1]} c\left(Q_{u}^{m}\right)<1$. We will still denote by $\|\cdot\|_{T V}$ the total variation distance (or the total variation norm if we consider the space of signed measures) on $\mathcal{P}\left(E^{j}\right)$ for any integer $j$. Moreover, let $\left(X_{k}(u)\right)_{k \in \mathbb{Z}}$ be a stationary Markov chain with transition $Q_{u}$, for $u \in[0,1]$. We remind that for an integer $j \geq 1$, $\pi_{k, j}^{(n)}\left(\right.$ resp. $\left.\pi_{u, j}\right)$ denotes the probability distribution of the vector $\left(X_{n, k}, \ldots, X_{n, k+j-1}\right)$ (resp. of the vector $\left.\left(X_{k}(u), \ldots, X_{k+j-1}(u)\right)\right)$,

Theorem 1. Assume that assumptions $\mathbf{A 1}$ - A2 hold true. Then for all $u \in[0,1]$, the Markov kernel $Q_{u}$ has a single invariant probability $\pi_{u}$. The triangular array of Markov chain $\left\{X_{n, k}, n \in \mathbb{Z}^{+}, k \leq n\right\}$ is locally stationary. Moreover, there exists a positive real number $C$, only depending on $L, m, r, \kappa$ such that

$$
\left\|\pi_{k, j}^{(n)}-\pi_{u, j}\right\|_{T V} \leq C\left[\sum_{s=k}^{k+j-1}\left|u-\frac{s}{n}\right|^{\kappa}+\frac{1}{n^{k}}\right] .
$$

Note. Assumption $\mathbf{A 1}$ is satisfied if there exist a positive real number $\varepsilon$, a positive integer $m$ and a family of probability measures $\left\{v_{u}: u \in[0,1]\right\}$ such that

$$
Q_{u}^{m}(x, A) \geq \varepsilon v_{u}(A), \text { for all }(u, x, A) \in[0,1] \times E \times \mathcal{B}(E) .
$$

In the homogeneous case, this condition is the so-called Doeblin's condition (see Meyn and Tweedie (2009), Chapter 16 for a discussion about this condition). To show that this condition is sufficient for A1, one can use the inequalities

$$
Q_{u}^{m}(x, A)-Q_{u}^{m}(y, A) \leq 1-\varepsilon+\varepsilon v_{u}(E \backslash A)-Q_{u}^{m}(x, E \backslash A) \leq 1-\varepsilon .
$$

For a Markov chain with a finite state space, the Doeblin's condition is satisfied if $\inf _{u \in[0,1]} Q_{u}^{m}(x, y)>0$, taking the counting measure for $v_{u}$. More generally, this condition is satisfied if $Q_{u}(x, A)=\int_{A} f_{u}(x, y) v(d y)$ with a probability measure $v$ and a density uniformly lower bounded, i.e $\varepsilon=\inf _{(u, x, y) \in[0,1] \times E^{2}} f_{u}(x, y)>0$.

Proof of Theorem 1 We remind that for a Markov kernel $R$ on $(E, \mathcal{E})$ and $\mu, v \in \mathcal{P}(E)$, we have

$$
\|\mu R-v R\|_{T V} \leq c(R) \cdot\|\mu-v\|_{T V},
$$

where $c(R)=\sup _{(x, y) \in E}\left\|\delta_{x} R-\delta_{y} R\right\|_{T V} \in[0,1]$. Then, under our assumptions, the application $T: \mathcal{P}(E) \rightarrow$ $\mathcal{P}(E)$ defined by $T(\mu)=\mu Q_{u}^{m}$ is contractant and the existence and uniqueness of an invariant probability $\pi_{u}$ easily follows from the fixed point theorem in a complete metric space.

We next show Condition 1 of Definition 1 . The result is shown by induction. For $j=1$, we have from assumption A1,

$$
\begin{aligned}
\left\|\pi_{u}-\pi_{v}\right\|_{T V} & \leq\left\|\pi_{u} Q_{u}^{m}-\pi_{v} Q_{u}^{m}\right\|_{T V}+\left\|\pi_{v} Q_{u}^{m}-\pi_{v} Q_{v}^{m}\right\|_{T V} \\
& \leq r\left\|\pi_{u}-\pi_{v}\right\|_{T V}+\sup _{x \in E}\left\|\delta_{x} Q_{u}^{m}-\delta_{x} Q_{v}^{m}\right\|_{T V} .
\end{aligned}
$$

Since for two Markov kernels $R$ and $\widetilde{R}$ and $\mu, v \in \mathcal{P}(E)$, we have

$$
\|\mu R-v \widetilde{R}\|_{T V} \leq \sup _{x \in E}\left\|\delta_{x} R-\delta_{x} \widetilde{R}\right\|_{T V}+c(\widetilde{R})\|\mu-v\|_{T V},
$$


we deduce from assumption $\mathbf{A 2}$ that $\sup _{x \in E}\left\|\delta_{x} Q_{u}^{m}-\delta_{x} Q_{v}^{m}\right\|_{T V} \leq m L|u-v|^{\kappa}$. This leads to the inequality

$$
\left\|\pi_{u}-\pi_{v}\right\|_{T V} \leq \frac{m L}{1-r}|u-v|^{\kappa}
$$

which shows the result for $j=1$. If the continuity condition holds true for $j-1$, we note that

$$
\pi_{u, j}\left(d x_{1}, \ldots, d_{x_{j-1}}\right)=\pi_{u, j-1}\left(d x_{1}, \ldots, d_{x_{j-1}}\right) Q_{u}\left(x_{j-1}, d x_{j}\right)
$$

Moreover, we have

$$
\left\|\pi_{u, j}-\pi_{v, j}\right\|_{T V} \leq \sup _{x \in E}\left\|\delta_{x} Q_{u}-\delta_{x} Q_{v}\right\|_{T V}+\left\|\pi_{u, j-1}-\pi_{v, j-1}\right\|_{T V},
$$

which leads to the continuity of $u \mapsto \pi_{u, j}$. This justifies Condition 1 of Definition 1 .

Finally we prove the bound announced for $\left\|\pi_{k, j}^{(n)}-\pi_{u, j}\right\|_{T V}$. Note that this bound automatically implies Condition 2 of Definition 1. Let us first note that if $R_{k, m}=Q_{\frac{k-m+1}{n}} Q_{\frac{k-m+2}{n}} \cdots Q_{\frac{k}{n}}$, we have from assumption A2,

$$
\sup _{x \in E}\left\|\delta_{x} R_{k, m}-\delta_{x} Q_{u}^{m}\right\|_{T V} \leq L \sum_{s=k-m+1}^{k}\left|u-\frac{s}{n}\right|^{K} .
$$

Now for $j=1$, we have

$$
\begin{aligned}
\left\|\pi_{k}^{(n)}-\pi_{u}\right\|_{T V} & \leq\left\|\pi_{k-m}^{(n)} R_{k, m}-\pi_{k-m}^{(n)} Q_{u}^{m}\right\|_{T V}+\left\|\pi_{k-m}^{(n)} Q_{u}^{m}-\pi_{u} Q_{u}^{m}\right\|_{T V} \\
& \leq L \sum_{s=k-m+1}^{k}\left|u-\frac{s}{n}\right|^{K}+r\left\|\pi_{k-m}^{(n)}-\pi_{u}\right\|_{T V} .
\end{aligned}
$$

Using the fact that is $s \leq 0,|u-s / n| \leq|u|$, we deduce that

$$
\left\|\pi_{k}^{(n)}-\pi_{u}\right\|_{T V} \leq L \sum_{\ell=0}^{\infty} r^{\ell} \sum_{s=k-(\ell+1) m+1}^{k-\ell m}\left|u-\frac{s}{n}\right|^{K},
$$

which shows the result for $j=1$. Next, using the same argument as for the continuity of the finitedimensional distributions, we have

$$
\left\|\pi_{k, j}^{(n)}-\pi_{u, j}\right\|_{T V} \leq L\left|u-\frac{k+j-1}{n}\right|^{K}+\left\|\pi_{k, j-1}^{(n)}-\pi_{u, j-1}\right\|_{T V} .
$$

Hence the result easily follows by iteration.

\section{$2.2 \beta$-mixing properties}

In this subsection, we consider the problem of mixing for the locally stationary Markov chains introduced previously. For convenience, we assume that $X_{n, j}$ is equal to zero if $j \geq n+1$. For a positive integer $n$ and an integer $i \in \mathbb{Z}$, we denote by $\mathcal{F}_{i}^{(n)}$ the sigma field $\sigma\left(X_{n, j}: j \leq i\right)$. Now setting

$$
V\left(\mathcal{F}_{i}^{(n)}, X_{n, i+j}\right)=\sup \left\{\left|\mathbb{E}\left[f\left(X_{n, i+j} \mid \mathcal{F}_{i}^{(n)}\right)\right]-\mathbb{E}\left[f\left(X_{n, i+j}\right)\right]\right|: \quad f \text { s.t }\|f\|_{\infty} \leq 1\right\},
$$

the $\beta_{n}$-mixing coefficient for the sequence $\left(X_{n, j}\right)_{j \in \mathbb{Z}}$ is defined by

$$
\beta_{n}(j)=\frac{1}{2} \sup _{i \in \mathbb{Z}} \mathbb{E}\left[V\left(\mathcal{F}_{i}^{(n)}, X_{n, i+j}\right)\right] .
$$

Under our assumptions, this coefficient is shown to decrease exponentially fast. 
Proposition 1. Assume that assumptions A1 - A2 hold true. Then there exist $C>0$ and $\rho \in(0,1)$, only depending on $m, L, \kappa$ and $r$ such that

$$
\beta_{n}(j) \leq C \rho^{[j / m]},
$$

where $[x]$ denotes the integer part of a real number $x$.

Note. The usual strong mixing coefficient is defined for Markov chains by

$$
\alpha_{n}(j)=\sup _{i \in \mathbb{Z}}\left\{|\mathbb{P}(A \cap B)-\mathbb{P}(A) \mathbb{P}(B)|: A \in \sigma\left(X_{n, i}\right), B \in \sigma\left(X_{n, i+j}\right)\right\} .
$$

We have $\alpha_{n}(j) \leq \beta_{n}(j)$. We refer the reader Doukhan (1994) for the definition of some classical mixing coefficients and their properties. In this paper, we will mainly use some results available for the larger class of strong-mixing processes.

Proof of Proposition 1 We first consider $\epsilon>0$ such that $\rho=2 m L \epsilon^{k}+r<1$. Assume first that $n \geq \frac{m}{\epsilon}$. For $k \leq n$, we set $Q_{k, m}=Q_{\frac{k-m+1}{n}} \cdots Q_{\frac{k}{n}}$. By noticing that under Assumption A2, we have

$$
\sup _{\mu \in \mathcal{P}(E)}\left\|\mu Q_{u}-\mu Q_{v}\right\|_{T V} \leq L|u-v|^{K},
$$

we deduce the bound

$$
\sup _{x \in E}\left\|\delta_{x} Q_{k, m}-\delta_{x} Q_{\frac{k}{n}}^{m}\right\|_{T V} \leq m L \epsilon^{k}
$$

Then, from Assumption A1, we get

$$
\sup _{x, y \in E}\left\|\delta_{x} Q_{k, m}-\delta_{y} Q_{k, m}\right\|_{T V} \leq \rho .
$$

Now if $j=t m+s$ for two positive integers $t, s$, we get

$$
\left\|\delta_{X_{n, k-j}} Q_{\frac{k-j+1}{n}} \cdots Q_{\frac{k}{n}}-\pi_{k-j}^{(n)} Q_{\frac{k-j+1}{n}} \cdots Q_{\frac{k}{n}}\right\|_{T V} \leq \rho^{t} .
$$

Now, if $n<\frac{m}{\epsilon}$, one can show that $\beta_{n}(j) \leq 1$, if $j \leq n$ and $\beta_{n}(j) \leq r^{\left[\frac{j-n}{m}\right]}$ if $j>n$. This leads to the result with an appropriate choice of $C$, e.g $C=\rho^{-\frac{1}{\epsilon}-1}$

\subsection{Finite state space Markov chains}

Let $E$ be a finite set. In this case, we obtain the following result.

Corollary 1. Let $\left\{Q_{u}: u \in[0,1]\right\}$ be a family of transition matrices such that for each $u \in[0,1]$, the Markov chain with transition matrix $Q_{u}$ is irreducible and aperiodic. Assume further that for all $(x, y) \in E^{2}$, the application $u \rightarrow Q_{u}(x, y)$ is $\kappa-H o ̈ l d e r$ continuous. Then Theorem 3 applies and the $\beta$-mixing coefficients are bounded as in Proposition 1 . 
Proof of Corollary1 Using the fact that

$$
\left\|\delta_{x} Q_{u}^{m}-\delta_{y} Q_{u}^{m}\right\|_{T V}=1-\sum_{z \in E} Q_{u}^{m}(x, z) \wedge Q_{u}^{m}(y, z) \leq 1-|E| \cdot \inf _{x, y \in E} Q_{u}^{m}(x, y)
$$

Then assumption $\mathbf{A 1}$ is satisfied as soon as $\inf _{u \in[0,1],(x, y) \in E^{2}} Q_{u}^{m}(x, y)>0$. From aperiodicity and irreducibility, it is well know that for each $u \in[0,1]$,

$$
m_{u}=\inf \left\{k \geq 1: \min _{(x, y) \in E^{2}} Q_{u}^{k}(x, y)>0\right\}<\infty .
$$

By continuity, the sets $O_{u}=\left\{v \in[0,1]: P_{v}^{m_{u}}>0\right\}$ are open subsets of [0,1]. From the compactness of the interval $[0,1],[0,1]$ can be covered by finitely many $O_{u}$, say $O_{u_{1}}, \ldots, O_{u_{d}}$. Then assumption $\mathbf{A} \mathbf{1}$ is satisfied with $m=\max _{1 \leq i \leq d} m_{u_{i}}$. Assumption $\mathbf{A 2}$ is automatically satisfied and Theorem 3 applies.

Now, we show that our results can be used for nonparametric kernel estimation of the invariant probability $\pi_{u}$ or the transition matrix $Q_{u}$. This kind of estimation requires an estimation of quantities of type $h_{u}=\mathbb{E}\left[f\left(X_{1}(u), \ldots, X_{\ell}(u)\right)\right]$ where $f: E^{\ell} \rightarrow \mathbb{R}$ is a function and $\ell$ is an integer. To this end, a classical method used for locally stationary time series is based on kernel estimation. See for instance Dahlhaus and Subba Rao (2006), Fryzlewicz et al. (2008), Vogt (2012) or Zhang and Wu (2015) for nonparametric kernel estimation of locally stationary processes. Let $K: \mathbb{R} \rightarrow \mathbb{R}_{+}$be a Lipschitz function supported on $[-1,1]$ and such that $\int K(z) d z=1$. For $b=b_{n} \in(0,1)$, we set

$$
e_{i}(u)=\frac{\frac{1}{n b} K\left(u-\frac{i}{n}\right)}{\frac{1}{n b} \sum_{j=\ell}^{n} K\left(u-\frac{j}{n}\right)}, \quad u \in[0,1], \quad \ell \leq i \leq n .
$$

A natural estimator of $h_{u}$ is

$$
\hat{h}_{u}=\sum_{i=\ell}^{n} e_{i}(u) f\left(X_{n, i-\ell+1}, \ldots, X_{n, i}\right) .
$$

The next proposition gives a uniform control of the variance part $\hat{h}_{u}-\mathbb{E} \hat{h}_{u}$.

Proposition 2. Assume that assumption $\mathbf{A 3}$ holds true and that $b \rightarrow 0, n b^{1+\epsilon} \rightarrow \infty$ for some $\epsilon>0$. Then

$$
\sup _{u \in[0,1]}\left|\hat{h}_{u}-\mathbb{E} \hat{h}_{u}\right|=O_{\mathbb{P}}\left(\frac{\sqrt{\log n}}{\sqrt{n b}}\right) .
$$

Proof of Proposition 2 We set $Y_{n, i}=f\left(X_{n, i-\ell+1}, \ldots, X_{n, i}\right)$. First, note that the triangular array $\left(Y_{n, i}\right)_{1 \leq i \leq n}$ is $\beta$-mixing (and then $\alpha$-mixing) with $\beta_{n}(j) \leq \widetilde{C} \rho^{\left[\frac{j-\ell}{m}\right]} \leq \widetilde{C} \rho^{-1-\frac{\ell}{m}} \widetilde{\rho}^{j}$ where $\widetilde{C}$ is a positive constant and $\widetilde{\rho}=\rho^{1 / m}$. We have $[0,1]=\cup_{s=1}^{k+1} I_{s}$ where $k$ is the integer part of $1 / b, I_{s}=((s-1) b, s b]$ for $1 \leq s \leq k$ and $I_{k+1}=(k b, 1]$. We set $S_{0}^{(n)}=0$ and if $1 \leq i \leq n, S_{i}^{(n)}=\sum_{s=1}^{i} Z_{s}^{(n)}$, where $Z_{s}^{(n)}=Y_{n, s}-\mathbb{E} Y_{n, s}$. Then for $1 \leq j \leq j+k \leq n$, we have

$$
\begin{aligned}
\left|\sum_{i=j}^{j+k} e_{i}(u) Z_{i}^{(n)}\right| & \leq e_{j}(u) \cdot\left|S_{j-1}^{(n)}\right|+e_{j+k}(u) \cdot\left|S_{j+k}^{(n)}\right|+\sum_{i=j}^{j+k-1}\left|e_{i}(u)-e_{i-1}(u)\right| \cdot\left|S_{i}^{(n)}\right| \\
& \leq \frac{C^{\prime \prime}}{n b} \max _{j-1 \leq i \leq j+k}\left|S_{i}^{(n)}\right| .
\end{aligned}
$$


This gives the bound

$$
\begin{aligned}
\max _{u \in[0,1]}\left|\sum_{i=1}^{n} e_{i}(u) Z_{i}^{(n)}\right| & \leq \max _{1 \leq s \leq k} \max _{u \in I_{s}}\left|\sum_{n(s-2) b \leq i \leq n(s+1) b} e_{i}(u) Z_{i}^{(n)}\right| \\
& \leq \frac{C^{\prime \prime}}{n b} \max _{1 \leq s \leq k+1} \max _{n(s-2) b-1 \leq i \leq n(s+1) b}\left|S_{i}^{(n)}\right| .
\end{aligned}
$$

We will use the exponential inequality for strong mixing sequences given in Rio (1999), Theorem 6.1 (see also Rio (2013), Theorem 6.1). This inequality guarantees that for any integer $q$, we have

$$
\mathbb{P}\left(\max _{n(s-2) b-1 \leq i \leq n(s+1) b}\left|S_{i}^{(n)}\right| \geq F \lambda\right) \leq G \exp \left(-\frac{\lambda}{2 q\|f\|_{\infty}} \log \left(1+K \frac{\lambda q}{n b}\right)\right)+M n b \frac{\widetilde{\rho}^{q}}{\lambda},
$$

where $F, G, K, M$ are three positive real numbers not depending on $n$ and $s$ and $\lambda \geq q\|f\|_{\infty}$. We have $k=O\left(b^{-1}\right)$ and setting $q \approx \frac{\sqrt{n b}}{\sqrt{\log n}}$ and $\lambda=\lambda^{\prime} \sqrt{n b \log n}$, we have for $\lambda^{\prime}$ large enough

$$
\mathbb{P}\left(\max _{u \in[0,1]}\left|\sum_{i=1}^{n} e_{i}(u) Z_{i}^{(n)}\right|>\frac{F \lambda}{n b}\right)=O_{\mathbb{P}}\left(\frac{1}{b n^{\frac{1}{1+\epsilon}}}+\frac{\sqrt{n b}}{b \sqrt{\log (n)}} \widetilde{\rho}^{\frac{\sqrt{n b}}{\log (n)}}\right) .
$$

Then the result follows from the bandwidth conditions. $\square$

Now, we consider some estimators of $\pi_{u}$ and $Q_{u}$. Let $\hat{\pi}_{u}(x)=\sum_{i=1}^{n-1} e_{i}(u) \mathbb{1}_{X_{n, i}=x}$ and $\hat{Q}_{u}(x, y)=\frac{\hat{\pi}_{u, 2}(x, y)}{\hat{\pi}_{u}(x)}$. where $\hat{\pi}_{u, 2}(x, y)=\sum_{i=1}^{n-1} e_{i}(u) \mathbb{1}_{X_{n, i}=x, X_{n, i+1}=y}$.

Theorem 2. Assume that for a given $\epsilon>0, b \rightarrow 0$ and $n b^{1+\epsilon} \rightarrow \infty$.

1. For $(x, y) \in E^{2}$, we have

$$
\sup _{u \in[0,1]}\left|\mathbb{E} \hat{\pi}_{u}(x)-\pi_{u}(x)\right|=O\left(b^{\kappa}\right), \sup _{u \in[0,1]}\left|\frac{\mathbb{E} \hat{\pi}_{u, 2}(x, y)}{\mathbb{E} \hat{\pi}_{u}(x)}-Q_{u}(x, y)\right|=O\left(b^{\kappa}\right)
$$

and For $(x, y) \in E^{2}$, we have

$$
\sup _{u \in[0,1]}\left|\hat{\pi}_{u}(x)-\mathbb{E} \hat{\pi}_{u}(x)\right|=O\left(\frac{\sqrt{\log (n)}}{\sqrt{n b}}\right), \quad \sup _{u \in[0,1]}\left|\hat{Q}_{u}(x, y)-\frac{\mathbb{E} \hat{\pi}_{u, 2}(x, y)}{\mathbb{E} \hat{\pi}_{u}(x)}\right|=O\left(\frac{\sqrt{\log (n)}}{\sqrt{n b}}\right) .
$$

2. For $(u, x) \in[0,1] \times E$, the vector $\left(\sqrt{n b}\left[\hat{\pi}_{u}(x)-\mathbb{E} \hat{\pi}_{u}(x)\right]\right)_{x \in E}$ is asymptotically Gaussian with mean 0 and covariance $\Sigma_{u}^{(1)}: E \times E \rightarrow \mathbb{R}$ defined by

$$
\Sigma_{u}^{(1)}=\int K^{2}(x) d x \cdot\left[\Gamma_{u}(0)+\sum_{j \geq 1}\left(\Gamma_{u}(j)+\Gamma_{u}(j)^{\prime}\right)\right],
$$

where $\Gamma_{u}(j)_{x, y}=\pi_{u}(x) Q_{u}^{j}(x, y)-\pi_{u}(x) \pi_{u}(y)$.

3. For $(u, x, y) \in[0,1] \times E^{2}$, the vector

$$
\sqrt{n b}\left(\hat{Q}_{u}(x, y)-\frac{\mathbb{E} \hat{\pi}_{u, 2}(x, y)}{\mathbb{E} \hat{\pi}_{u}(x)}\right)_{(x, y) \in E^{2}}
$$

is asymptotically Gaussian with mean 0 and covariance $\Sigma^{(2)}: E^{2} \times E^{2} \rightarrow \mathbb{R}$ defined by

$$
\Sigma_{u}^{(2)}\left((x, y),\left(x^{\prime}, y^{\prime}\right)\right)=\int K^{2}(x) d x \cdot \frac{1}{\pi_{u}(x)} Q_{u}(x, y)\left[\mathbb{1}_{y=y^{\prime}}-Q_{u}\left(x^{\prime}, y^{\prime}\right)\right] \mathbb{1}_{x=x^{\prime}} .
$$


Note. Our estimators are localized versions of the standard estimators used in the homogeneous case. One can see that their convergence rates are standard for nonparametric kernel estimation.

\section{Proof of Theorem 2}

1. For the control of the bias, note that

$$
\mathbb{E} \hat{\pi}_{u, 2}(x, y)-\pi_{u, 2}(x, y)=\sum_{i=1}^{n-1} e_{i}(u)\left[\pi_{i, 2}^{(n)}(x, y)-\pi_{u, 2}(x, y)\right] .
$$

Since $e_{i}(u)=0$ if $|u-i / n|>b$, Theorem 1 ensures that

$$
\sup _{u \in[0,1]}\left|\mathbb{E} \hat{\pi}_{u, 2}(x, y)-\pi_{u, 2}(x, y)\right|=O\left(b^{K}+\frac{1}{n^{K}}\right)=O\left(b^{K}\right) .
$$

By summation on $y$, we deduce the first bound in (1) and using the fact that $\min _{u \in[0,1]} \pi_{u}(x)>0$, we deduce that $\max _{u \in[0,1]} \frac{1}{\mathbb{E} \hat{\pi}_{u}(x)}=O_{\mathbb{P}}(1)$ and the second bound in (1) follows.

For the variance terms in (2), we use Proposition 2 which ensures the first bound as well as $\max _{u \in[0,1]} \frac{1}{\hat{\pi}_{u}(x)}=$ $O_{\mathbb{P}}(1)$. This gives also the second bound.

2. The proof is based on a central limit theorem for triangular arrays of strongly mixing random variables proved in Rio (1995). This result is given in Proposition 8. For simplicity of notations, we consider the quantity $\sum_{i=1}^{n} e_{i}(u) \mathbb{1}_{X_{n, i}=x}$ instead of $\hat{\pi}_{u}(x)$ which has the same asymptotic behavior. For $x \in E$, let $\lambda_{x}$ be a real number. We consider the random variables $Z_{i}^{(n)}=\sum_{x \in E} \lambda_{x} \mathbb{1}_{X_{n, i}=x}$ and $Z_{i}(u)=\sum_{x \in E} \lambda_{x} \mathbb{1}_{X_{i}(u)=x}$ and set

$$
G_{i}^{(n)}=\sqrt{n b} e_{i}(u)\left(Z_{i}^{(n)}-\mathbb{E} Z_{i}^{(n)}\right), \quad H_{i}^{(n)}=G_{i}^{(n)} / \sqrt{\operatorname{Var}\left(\sum_{j=1}^{n} G_{j}^{(n)}\right)} .
$$

Let us first derive the limit of $\operatorname{Var}\left(\sum_{j=1}^{n} G_{j}^{(n)}\right)$. Using Proposition 1, we know that there exists a constant $D>0$ and $\widetilde{\rho} \in(0,1)$, such that

$$
\left|\operatorname{Cov}\left(Z_{i}^{(n)}, Z_{j}^{(n)}\right)\right| \leq D \widetilde{\rho}^{|i-j|}
$$

Moreover the same type of inequality holds for $\operatorname{Cov}\left(Z_{i}(u), Z_{j}(u)\right)$. Then if $\ell$ is a positive integer, let $V_{n}(\ell)=\left\{(i, j) \in\{1,2, \ldots, n\}^{2}:|i-j| \leq \ell\right\}$. We have

$$
\begin{aligned}
\operatorname{Var}\left(\sum_{j=1}^{n} G_{j}^{(n)}\right) & =\sum_{(i, j) \in V_{n}(\ell)} \operatorname{Cov}\left(G_{i}^{(n)}, G_{j}^{(n)}\right)+\sum_{(i, j) \in\{1, \ldots, n\}^{2} \backslash V_{n}(\ell)} \operatorname{Cov}\left(G_{i}^{(n)}, G_{j}^{(n)}\right) \\
& =A_{1}+A_{2} .
\end{aligned}
$$

If $G_{i}(u)=\sqrt{n b} e_{i}(u)\left(Z_{i}(u)-\mathbb{E} Z_{i}(u)\right)$, we can also decompose

$$
\begin{aligned}
\operatorname{Var}\left(\sum_{j=1}^{n} G_{j}(u)\right) & =\sum_{(i, j) \in V_{n}(\ell)} \operatorname{Cov}\left(G_{i}(u), G_{j}(u)\right)+\sum_{(i, j) \in\{1, \ldots, n\}^{2} \backslash V_{n}(\ell)} \operatorname{Cov}\left(G_{i}(u), G_{j}(u)\right) \\
& =A_{1}(u)+A_{2}(u) .
\end{aligned}
$$


Using (3), we have

$$
\begin{aligned}
\left|A_{2}\right| & \leq 2 n b D \sum_{i=1}^{n} e_{i}(u) \sum_{j=i+\ell+1}^{n} e_{j}(u) \widetilde{\rho}^{j-i} \\
& \leq 2 n b D \max _{1 \leq j \leq n} e_{j}(u) \frac{\widetilde{\rho}^{\ell}}{1-\rho} . \\
& =O\left(\tilde{\rho}^{\ell}\right) .
\end{aligned}
$$

In the same way, $\left|A_{2}(u)\right|=O\left(\widetilde{\rho}^{\ell}\right)$. Moreover, using Theorem 1, we have

$$
\begin{aligned}
\left|A_{1}-A_{1}(u)\right| & \leq 2 C n b \sum_{i=1}^{n} e_{i}(u) \sum_{j=i}^{(i+\ell) \wedge n} e_{j}(u)\left[\sum_{s=i}^{j}\left|u-\frac{s}{\ell}\right|^{K}+\frac{1}{n^{K}}\right] \\
& \leq 4 C n b \max _{1 \leq j \leq n} e_{j}(u)\left[(\ell+1)^{2} b^{K}+\frac{\ell+1}{n^{K}}\right] \\
& =O\left(\ell^{2} b^{\kappa}+\frac{\ell}{n^{K}}\right) .
\end{aligned}
$$

Then, choosing $\ell=\ell_{n}$ such that $\ell \rightarrow \infty, \ell^{2} b^{K} \rightarrow 0$ and $\ell / n^{K} \rightarrow 0$, we deduce that

$$
\operatorname{Var}\left(\sum_{j=1}^{n} G_{j}^{(n)}\right)=\operatorname{Var}\left(\sum_{j=1}^{n} G_{j}(u)\right)+o(1) .
$$

Now, we have

$$
\begin{aligned}
\operatorname{Var}\left(\sum_{j=1}^{n} G_{j}(u)\right) & =n b \sum_{i=1}^{n} e_{i}(u)^{2} \operatorname{Var}\left(Z_{0}(u)\right) \\
& +2 n b \sum_{i=1}^{n} \sum_{j=i+1}^{n} e_{i}(u) e_{j}(u) \operatorname{Cov}\left(Z_{0}(u), Z_{j-i}(u)\right) \\
& =n b \sum_{i=1}^{n} e_{i}(u)^{2} \operatorname{Var}\left(Z_{0}(u)\right) \\
& +2 n b \sum_{s=1}^{n-1}\left[\sum_{i=1}^{n-s} e_{i}(u) e_{i+s}(u)\right] \sum_{x, y \in E} \lambda_{x} \lambda_{y} \Gamma_{u}(s)_{x, y} .
\end{aligned}
$$

Using the Lebesgue theorem and elementary computations with Riemanian sums involving the kernel, we deduce that

$$
\lim _{n \rightarrow \infty} \operatorname{Var}\left(\sum_{j=1}^{n} G_{j}(u)\right)=\sum_{x, y \in E} \lambda_{x} \lambda_{y} \Sigma_{u, x, y}^{(1)} .
$$

Using (4), we also deduce that

$$
\lim _{n \rightarrow \infty} \operatorname{Var}\left(\sum_{j=1}^{n} G_{j}^{(n)}\right)=\sum_{x, y \in E} \lambda_{x} \lambda_{y} \Sigma_{u, x, y}^{(1)} .
$$


Next, in order to apply Proposition 8, we first check condition (13). We have $V_{n, n}=1$ and

$$
V_{n, i}=n b \sum_{s, t=1}^{i} e_{s}(u) e_{t}(u) \operatorname{Cov}\left(Z_{s}^{(n)}, Z_{t}^{(n)}\right) \leq n b \sum_{s, t=1}^{n} e_{s}(u) e_{t}(u)\left|\operatorname{Cov}\left(Z_{s}^{(n)}, Z_{t}^{(n)}\right)\right|=O(1),
$$

using (3). This entails condition (13) of Proposition 8. Finally, we check condition (14) of Proposition 8. From (5), we have $\max _{1 \leq i \leq n}\left|H_{i}^{(n)}\right| \leq C \mathbb{1}_{u-n b \leq i \leq u+n b} / \sqrt{n b}$ for a non random real number $C$ which does not depend on $n$. Then we have also $Q_{n, i}(x) \leq C \mathbb{1}_{u-n b \leq i \leq u+n b} / \sqrt{n b}$. Moreover, $\alpha_{(n)}(x)$ is bounded by (up to a constant) $-\log (x)+1$. This entails that

$$
V_{n, n}^{-3 / 2} \sum_{i=1}^{n} \int_{0}^{1} \alpha_{(n)}^{-1}(x / 2) Q_{n, i}^{2}(x) \inf \left(\alpha_{(n)}^{-1}(x / 2) Q_{n, i}(x), \sqrt{V_{n, n}}\right) d x=O\left(\frac{1}{\sqrt{n b}}\right) .
$$

Then we deduce the result of point 2 from Proposition 8, (5) and the Cramér-Wold device.

3. Let

$$
Z_{n}(x, y)=\frac{\sqrt{n b}}{\hat{\pi}_{u}(x)} \sum_{i=1}^{n-1} D_{n, i}(x, y)
$$

where

$$
D_{n, i}(x, y)=e_{i}(u)\left[\mathbb{1}_{X_{n, i}=x, X_{n, i+1}=y}-Q_{\frac{i+1}{n}}(x, y) \mathbb{1}_{X_{n, i}=x}\right]
$$

is a martingale increment bounded by $(n b)^{-1}$ (up to a constant). Using the classical Lindeberg central limit theorem for martingales, the sum $\sqrt{n b} \sum_{i=1}^{n-1}\left[D_{n, i}(x, y)\right]_{x, y \in E}$ is asymptotically a Gaussian vector with mean 0 and variance matrix $\Sigma$ defined by

$$
\begin{aligned}
& \sum\left((x, y),\left(x^{\prime}, y^{\prime}\right)\right) \\
= & \lim _{n \rightarrow \infty} n b \sum_{i=1}^{n-1} e_{i}(u)^{2} \operatorname{Cov}\left[\mathbb{1}_{X_{n, i}=x, X_{n, i+1}=y}-Q_{\frac{i+1}{n}}(x, y) \mathbb{1}_{X_{n, i}=x}, \mathbb{1}_{X_{n, i}=x^{\prime}, X_{n, i+1}=y^{\prime}}-Q_{\frac{i+1}{n}}\left(x^{\prime}, y^{\prime}\right) \mathbb{1}_{X_{n, i}=x^{\prime}}\right] \\
= & \lim _{n \rightarrow \infty} n b \sum_{i=1}^{n-1} e_{i}(u)^{2} \operatorname{Cov}\left[\mathbb{1}_{X_{i}(u)=x, X_{i+1}(u)=y}-Q_{u}(x, y) \mathbb{1}_{X_{i}(u)=x}, \mathbb{1}_{X_{i}(u)=x^{\prime}, X_{i+1}(u)=y^{\prime}}-Q_{u}\left(x^{\prime}, y^{\prime}\right) \mathbb{1}_{X_{i}(u)=x^{\prime}}\right] \\
= & \int K^{2}(z) d z \cdot \mathbb{P}\left(X_{1}(u)=x, X_{2}(u)=y\right) \cdot\left[\mathbb{1}_{y=y^{\prime}}-Q_{u}\left(x^{\prime}, y^{\prime}\right)\right] \mathbb{1}_{x=x^{\prime}} .
\end{aligned}
$$

In the previous equalities, we have used Theorem 1, the continuity properties of the transition matrix and the limits

$$
\lim _{n \rightarrow \infty} \frac{1}{n b} \sum_{i=1}^{n-1} K\left(\frac{u-i / n}{b}\right)=\int K(z) d z=1, \quad \lim _{n \rightarrow \infty} \frac{1}{n b} \sum_{i=1}^{n-1} K^{2}\left(\frac{u-i / n}{b}\right)=\int K(z)^{2} d z .
$$

We deduce that the vector $\left[Z_{n}(x, y)\right]_{x, y \in E}$ is asymptotically Gaussian with mean zero and covariance matrix $\Sigma_{u}^{(2)}$.

Then it remains to show that for each $(x, y) \in E^{2}$,

$$
\sqrt{n b}\left[\sum_{i=1}^{n} e_{i}(u) \frac{\mathbb{1}_{X_{n, i}=x} Q_{\frac{i+1}{n}}(x, y)}{\hat{\pi}_{u}(x)}-\frac{\mathbb{E} \hat{\pi}_{u, 2}(x, y)}{\mathbb{E} \hat{\pi}_{u}(x)}\right]=o_{\mathbb{P}}(1) .
$$


To show (6), we use the decomposition

$$
\begin{aligned}
& \sqrt{n b}\left[\sum_{i=1}^{n-1} e_{i}(u) \frac{\mathbb{1}_{X_{n, i}=x} Q_{\frac{i+1}{n}}(x, y)}{\hat{\pi}_{u}(x)}-\frac{\mathbb{E} \hat{\pi}_{u, 2}(x, y)}{\mathbb{E} \hat{\pi}_{u}(x)}\right] \\
= & \frac{\sqrt{n b}}{\hat{\pi}_{u}(x)} \sum_{i=1}^{n-1} e_{i}(u)\left(\mathbb{1}_{X_{n, i}=x}-\pi_{i}^{(n)}(x)\right) \cdot\left(Q_{\frac{i+1}{n}}(x, y)-Q_{u}(x, y)\right) \\
+ & \sqrt{n b} \sum_{i=1}^{n-1} e_{i}(u) \pi_{i}^{(n)}(x)\left(Q_{\frac{i+1}{n}}(x, y)-Q_{u}(x, y)\right) \frac{\pi_{u}(x)-\hat{\pi}_{u}(x)}{\hat{\pi}_{u}(x) \pi_{u}(x)} \\
= & \frac{A_{n}}{\hat{\pi}_{u}(x)}+B_{n} \frac{\pi_{u}(x)-\hat{\pi}_{u}(x)}{\hat{\pi}_{u}(x) \pi_{u}(x)} .
\end{aligned}
$$

Since the kernel $K$ has a compact support and $u \mapsto Q_{u}(x, y)$ is $\kappa$-Hölder continuous, we have $B_{n}=$ $O\left(\sqrt{n b} b^{\kappa}\right)$. Moreover, using covariance inequalities, we have $\operatorname{Var}\left(A_{n}\right)=O\left(b^{2 \kappa}\right)$. Then (6) follows from $\hat{\pi}_{u}(x)-\pi_{u}(x)=O_{\mathbb{P}}\left(\frac{1}{\sqrt{n b}}\right)$ and $\frac{1}{\hat{\pi}_{u}(x)}=O_{\mathbb{P}}(1)$. The proof of point 3 is now complete.

\section{Contraction of Markov kernels using Wasserstein metrics}

In this section, we consider a Polish space $(E, d)$. For $p \geq 1$, we consider the set of probability measures on $(E, d)$ admitting a moment of order $p$ :

$$
\mathcal{P}_{p}(E)=\left\{\mu \in \mathcal{P}(E): \int d\left(x, x_{0}\right)^{p} \mu(d x)<\infty\right\} .
$$

Here $x_{0}$ is an arbitrary point in $E$. It is easily seen that the set $\mathcal{P}_{p}(E)$ does not depend on $x_{0}$.

The Wasserstein metric $W_{p}$ of order $p$ associated to $d$ is defined by

$$
W_{p}(\mu, v)=\inf _{\gamma \in \Gamma(\mu, v)}\left\{\int_{E \times E} d(x, y)^{p} d \gamma(x, y)\right\}^{1 / p}
$$

where $\Gamma(\mu, v)$ denotes the collection of all probability measures on $E \times E$ with marginals $\mu$ and $v$. We will say that $\gamma \in \Gamma(\mu, v)$ is an optimal coupling of $(\mu, v)$ if

$$
\left(\int d(x, y)^{p} \gamma(d x, d y)\right)^{1 / p}=W_{p}(\mu, v) .
$$

It is well-known that an optimal coupling always exist. See Villani (2009) for some properties of Wasserstein metrics.

In the sequel, we will use the following assumptions.

B1 For all $(u, x) \in[0,1] \times E, \delta_{x} Q_{u} \in \mathcal{P}_{p}(E)$.

B2 There exist a positive integer $m$ and two real numbers $r \in(0,1)$ and $C_{1} \geq 1$ such that for all $u \in[0,1]$ and all $x \in E$,

$$
W_{p}\left(\delta_{x} Q_{u}^{m}, \delta_{y} Q_{u}^{m}\right) \leq r d(x, y), \quad W_{p}\left(\delta_{x} Q_{u}, \delta_{y} Q_{u}\right) \leq C_{1} d(x, y) .
$$

B3 The family of transitions $\left\{Q_{u}: u \in[0,1]\right\}$ satisfies the following Hölder type continuity condition. There exist $\kappa \in(0,1]$ and $C_{2}>0$, such that for all $x \in E$ and all $u, v \in[0,1]$,

$$
W_{p}\left(\delta_{x} Q_{u}, \delta_{x} Q_{v}\right) \leq C_{2}\left(1+d\left(x, x_{0}\right)\right)|u-v|^{\kappa} .
$$


Note. If $R$ is a Markov kernel, the Dobrushin contraction coefficient is now defined by

$$
c(R)=\sup _{\substack{(x, y) \in E^{2} \\ x \neq y}} \frac{W_{p}\left(\delta_{x} R, \delta_{y} R\right)}{d(x, y)} .
$$

Thus Assumption $\mathbf{B 2}$ means that $\sup _{u \in[0,1]} c\left(Q_{u}\right)<\infty$ and $\sup _{u \in[0,1]} c\left(Q_{u}^{m}\right)<1$.

The following proposition shows that under these assumptions, the marginal distribution of the Markov chain with transition $Q_{u}$ converges exponentially fast to its unique invariant probability distribution which is in turn Hölder continuous with respect to $u$, in Wasserstein metric.

Proposition 3. Assume that assumptions B1-B3 hold true and set for an integer $j \geq 1$,

1. For all $u \in[0,1]$, the Markov chain of transition $Q_{u}$ has a unique invariant probability distribution denoted by $\pi_{u}$. Moreover for all initial probability distribution $\mu \in \mathcal{P}_{p}(E)$, we have for $n=m j+s$

$$
W_{p}\left(\mu Q_{u}^{n}, \pi_{u}\right) \leq C_{1}^{s} r^{j}\left[\left(\int d\left(x, x_{0}\right)^{p} \mu(d x)\right)^{1 / p}+\kappa_{2}\right],
$$

where $\kappa_{2}=\sup _{u \in[0,1]}\left(\int d\left(x, x_{0}\right)^{p} \pi_{u}(d x)\right)^{1 / p}$.

2. If $u, v \in[0,1]$, we have

$$
W_{p}\left(\pi_{u}, \pi_{v}\right) \leq \frac{C_{2}|u-v|^{\kappa}}{1-r}\left[m C_{1}^{m-1} \kappa_{2}+\sum_{j=0}^{m-1} C_{1}^{j} \kappa_{1}(m-j-1)\right],
$$

where $\kappa_{1}(j)=\sup _{u \in[0,1]}\left(\int d\left(x, x_{0}\right)^{p} Q_{u}^{j}\left(x_{0}, d x\right)\right)^{1 / p}$.

Proof of Proposition 3 We first show that the quantities $\kappa_{1}(j)$ are finite. We set $q_{j}=\left(\int\left(1+d\left(x, x_{0}\right)\right)^{p} Q_{0}^{j}\left(x_{0}, d x\right)\right)^{1 / p}$. If $j \geq 1$, we have, using Lemma 1 ,

$$
\begin{aligned}
& W_{p}\left(\delta_{x_{0}} Q_{u}^{j}, \delta_{x_{0}} Q_{0}^{j}\right) \\
\leq & W_{p}\left(\delta_{x_{0}} Q_{u}^{j}, \delta_{x_{0}} Q_{0}^{j-1} Q_{u}\right)+W_{p}\left(\delta_{x_{0}} Q_{0}^{j-1} Q_{u}, \delta_{x_{0}} Q_{0}^{j}\right) \\
= & C_{1} W_{p}\left(\delta_{x_{0}} Q_{u}^{j-1}, \delta_{x_{0}} Q_{0}^{j-1}\right)+C_{2}|u|^{\kappa} q_{j-1} .
\end{aligned}
$$

Then we obtain

$$
W_{p}\left(\delta_{x_{0}} Q_{u}^{j}, \delta_{x_{0}} Q_{0}^{j}\right) \leq C_{2} \sum_{s=0}^{j-1} C_{1}^{s} q_{j-s-1} .
$$

Then, using Lemma 3 for the function $f(x)=1+d\left(x, x_{0}\right)$, we get

$$
\kappa_{1}(j) \leq q_{j}+C_{2} \sum_{s=0}^{j-1} C_{1}^{s} q_{j-s-1}
$$


1. The existence and unicity of an invariant probability $\pi_{u} \in \mathcal{P}_{p}$ easily follows from the fixed point theorem for a contractant application in the complete metric space $\left(\mathcal{P}_{p}, W_{p}\right)$.

Before proving the geometric convergence, let us show that the quantity $\kappa_{2}$ is finite. We have, using Lemma 1,

$$
\begin{aligned}
W_{p}\left(\pi_{u}, \pi_{0}\right) & \leq W_{p}\left(\pi_{u} Q_{u}^{m}, \pi_{0} Q_{u}^{m}\right)+W_{p}\left(\pi_{0} Q_{u}^{m}, \pi_{0} Q_{0}^{m}\right) \\
& \leq r W_{p}\left(\pi_{u}, \pi_{0}\right)+\left(\int W_{p}^{p}\left(\delta_{x} Q_{u}^{m}, \delta_{x} Q_{0}^{m}\right) \pi_{0}(d x)\right)^{1 / p} .
\end{aligned}
$$

Using (7) and Lemma (1), we have

$$
\begin{aligned}
W_{p}\left(\delta_{x} Q_{u}^{m}, \delta_{x} Q_{0}^{m}\right) & \leq W_{p}\left(\delta_{x} Q_{u}^{m}, \delta_{x_{0}} Q_{u}^{m}\right)+W_{p}\left(\delta_{x_{0}} Q_{u}^{m}, \delta_{x_{0}} Q_{0}^{m}\right)+W_{p}\left(\delta_{x} Q_{0}^{m}, \delta_{x_{0}} Q_{0}^{m}\right) \\
& \leq 2 r d\left(x, x_{0}\right)+C_{2} \sum_{s=0}^{m-1} C_{1}^{j} q_{m-s-1} .
\end{aligned}
$$

From the previous bound, we easily deduce the existence of a real number $D>0$, not depending on $u$, such that $W_{p}\left(\pi_{u}, \pi_{0}\right) \leq \frac{D}{1-r}$. Then, using Lemma 3, we get

$$
\kappa_{2} \leq \frac{D}{1-r}+\left(\int d\left(x, x_{0}\right)^{p} \pi_{0}(d x)\right)^{1 / p}
$$

which is finite.

Now, the geometric convergence is a consequence of the inequality

$$
W_{p}\left(\mu Q_{u}^{n}, \pi_{u} Q_{u}^{n}\right) \leq C_{1}^{s} r^{j} W_{p}\left(\mu, \pi_{u}\right) \leq C_{1}^{s} r^{j}\left[\left(\int d\left(x, x_{0}\right)^{p} \mu(d x)\right)^{1 / p}+\kappa_{2}\right] .
$$

Finally, let $v$ be an invariant probability for $P_{u}$ (not necessarily in $\mathcal{P}_{p}$ ). Let $f: E \rightarrow \mathbb{R}$ be an element of $C_{b}(E)$. Since convergence in Wasserstein metric implies weak convergence, we have from the geometric ergodicity $\lim _{n \rightarrow \infty} Q_{u}^{n} f(x)=\pi_{u} f$ for all $x \in E$. Hence, using the Lebesgue theorem, we have

$$
v f=v Q_{u}^{n} f=\int v(d x) Q_{u}^{n} f(x) \rightarrow \pi_{u} f
$$

which shows the unicity of the invariant measure.

2. Proceeding as for the previous point, we have

$$
W_{p}\left(\pi_{u}, \pi_{v}\right) \leq r W_{p}\left(\pi_{u}, \pi_{v}\right)+\left(\int W_{p}^{p}\left(\delta_{x} Q_{u}^{m}, \delta_{x} Q_{v}^{m}\right) \pi_{v}(d x)\right)^{1 / p} .
$$

But

$$
\begin{aligned}
W_{p}\left(\delta_{x} Q_{u}^{m}, \delta_{x} Q_{v}^{m}\right) & \leq C_{1} W_{p}\left(\delta_{x} Q_{u}^{m-1}, \delta_{x} Q_{v}^{m-1}\right)+C_{2}|u-v|^{\kappa}\left(\int\left[1+d\left(y, x_{0}\right)\right]^{p} Q_{v}^{m-1}(x, d y)\right)^{1 / p} \\
& \leq C_{1} W_{p}\left(\delta_{x} Q_{u}^{m-1}, \delta_{x} Q_{v}^{m-1}\right)+C_{2}|u-v|^{\kappa}\left(\kappa_{1}(m-1)+C_{1}^{m-1} d\left(x, x_{0}\right)\right) .
\end{aligned}
$$


We deduce that

$$
W_{p}\left(\delta_{x} Q_{u}^{m}, \delta_{x} Q_{v}^{m}\right) \leq C_{2}|u-v|^{\kappa}\left(\sum_{j=0}^{m-1} C_{1}^{j} \kappa_{1}(m-j-1)+m C_{1}^{m-1} d\left(x, x_{0}\right)\right) .
$$

Reporting the last bound in (8), we get the result. $\square$

Now let us give the main result of this section. For $j \in \mathbb{N}^{*}$, we endow the space $E^{j}$ with the distance

$$
d_{j}(x, y)=\left(\sum_{s=1}^{j} d\left(x_{s}, y_{s}\right)^{p}\right)^{1 / p}, \quad x, y \in E^{j}
$$

We will still denote by $W_{p}$ the Wasserstein metric for Borelian measures on $E^{j}$.

Theorem 3. Assume that assumptions B1 - B3 hold true. Then the triangular array of Markov chains $\left\{X_{n, k}: n \in \mathbb{Z}^{+}, k \leq n\right\}$ is locally stationary. Moreover, there exists a real number $C>0$, only depending on $j, p, d, \kappa, r, C_{1}, C_{2}, \kappa_{1}(1), \ldots, \kappa_{1}(m), \kappa_{2}$ such that

$$
W_{p}\left(\pi_{k, j}^{(n)}, \pi_{u, j}\right) \leq C\left[\sum_{s=k}^{k+j-1}\left|u-\frac{s}{n}\right|^{\kappa}+\frac{1}{n^{\kappa}}\right] .
$$

\section{Proof of Theorem 3}

1. We show the result by induction and first consider the case $j=1$. For $k \leq n$, let $Q_{k, m}$ be the probability kernel $Q_{\frac{k-m+1}{n}} \cdots Q_{\frac{k}{n}}$. We have

$$
\begin{aligned}
W_{p}\left(\pi_{k}^{(n)}, \pi_{u}\right) & =W_{p}\left(\pi_{k-m}^{(n)} Q_{k, m}, \pi_{u} Q_{u}^{m}\right) \\
& \leq W_{p}\left(\pi_{k-m}^{(n)} Q_{k, m}, \pi_{k-m}^{(n)} Q_{u}^{m}\right)+W_{p}\left(\pi_{k-m}^{(n)} Q_{u}^{m}, \pi_{u} Q_{u}^{m}\right) \\
& \leq r W_{p}\left(\pi_{k-m}^{(n)}, \pi_{u}\right)+\left(\int W_{p}^{p}\left(\delta_{x} Q_{k, m}, \delta_{x} Q_{u}^{m}\right) \pi_{k-m}^{(n)}(d x)\right)^{1 / p} .
\end{aligned}
$$

From Lemma 2, we have

$$
W_{p}\left(\delta_{x} Q_{k, m}, \delta_{x} Q_{u}^{m}\right) \leq \sum_{s=0}^{m-1} C_{1}^{s} C_{2}\left[\int\left(1+d\left(y, x_{0}\right)\right)^{p} \delta_{x} Q_{\frac{k-m+1}{n}} \cdots Q_{\frac{k-s-1}{n}}(d y)\right]^{1 / p}\left|u-\frac{k-s}{n}\right|^{K} .
$$

First we note that from our assumptions and using Lemma 3 for the function $f(x)=1+d\left(x, x_{0}\right)$, we have

$$
\left[\delta_{x} Q_{u} f^{p}\right]^{1 / p} \leq\left[\delta_{x_{0}} Q_{u} f^{p}\right]^{1 / p}+C_{1} d\left(x, x_{0}\right) \leq\left(1+\kappa_{1}(1)+C_{1}\right) f(x),
$$

where $\kappa_{1}$ is defined in Proposition 3. Then we get $\sup _{u \in[0,1]} \delta_{x} Q_{u} f^{p} \leq C_{3}^{p} f^{p}(x)$, where $C_{3}=1+$ $\kappa_{1}(1)+C_{1}$. This yields to the inequality

$$
W_{p}\left(\delta_{x} Q_{k, m}, \delta_{x} Q_{u}^{m}\right) \leq \sum_{s=0}^{m-1} C_{1}^{s} C_{2} C_{3}^{m-s-1}\left|u-\frac{k-s}{n}\right|^{\kappa} f(x) .
$$


Then we obtain

$$
W_{p}\left(\pi_{k}^{(n)}, \pi_{u}\right) \leq r W_{p}\left(\pi_{k-m}^{(n)}, \pi_{u}\right)+\sum_{s=0}^{m-1} C_{1}^{s} C_{2} C_{3}^{m-s-1}\left|u-\frac{k-s}{n}\right|^{k}\left(\pi_{k-m}^{(n)} f^{p}\right)^{1 / p} .
$$

Then the result will easily follow if we prove that $\sup _{n, k \leq n} \pi_{k}^{(n)} f^{p}<\infty$. Setting $c_{k}=W_{p}\left(\pi_{k}^{(n)}, \pi_{\frac{k}{n}}\right)$ and $C_{4}=\sum_{s=0}^{m-1}(s+1)^{\kappa} C_{1}^{s+1} C_{2} C_{3}^{m-s-1}$ and using our previous inequality, we have

$$
\begin{aligned}
c_{k} & \leq r W_{p}\left(\pi_{k-m}^{(n)}, \pi_{\frac{k}{n}}\right)+\frac{C_{4}}{n^{\kappa}}\left(\pi_{k-m}^{(n)} f^{p}\right)^{1 / p} \\
& \leq\left(r+\frac{C_{4}}{n^{\kappa}}\right) c_{k-m}+r W_{p}\left(\pi_{\frac{k-m}{n}}, \pi_{\frac{k}{n}}\right)+\frac{C_{4}}{n^{\kappa}}\left(1+\kappa_{2}\right) .
\end{aligned}
$$

Then, if $n_{0}$ is such that for all $n \geq n_{0}, r+\frac{C_{4}}{n^{k}}<1$, the last inequality, Proposition 3 and Lemma 3 guarantee that $\sup _{n \geq n_{0}, k \leq n} \pi_{k}^{(n)} f^{p}$ is finite and only depends on $p, d, r, C_{1}, C_{2}, \kappa_{1}(1), \ldots, \kappa_{1}(m), \kappa_{2}, \kappa$. Moreover if $n \leq n_{0}$, we have $\left(\pi_{k}^{(n)} f^{p}\right)^{1 / p} \leq\left(C_{4}+1\right)^{n_{0}}\left(\pi_{0} f^{p}\right)^{1 / p}$. This concludes the proof for the case $j=1$.

2. Now for $j \geq 2$, we define a coupling of $\left(\pi_{k, j}^{(n)}, \pi_{u, j}\right)$ as follows. First we consider an optimal coupling $\Gamma_{u, j-1}^{(k, n)}$ of $\left(\pi_{k, j-1}^{(n)}, \pi_{u, j-1}\right)$, and for each $(x, y) \in E^{2}$, we define an optimal coupling $\Delta_{x, y, j, u}^{(k, n)}$ of $\left(\delta_{x} Q_{\frac{k+j}{n}}, \delta_{y} Q_{u}\right)$. From Villani (2009), Corollary 5.22, it is possible to choose this optimal coupling such that the application $(x, y) \mapsto \Delta_{x, y, j, u}^{(k, n)}$ is measurable. Now we define

$$
\Gamma_{u, j}^{(k, n)}\left(d x_{1}, d y_{1}, \ldots, d x_{j}, d y_{j}\right)=\Delta_{x_{j-1}, y_{j-1}, j, u}^{(k, n)}\left(d x_{j}, d y_{j}\right) \Gamma_{u, j-1}^{(k, n)}\left(d x_{1}, d y_{1}, \ldots, d x_{j-1}, d y_{j-1}\right) .
$$

Then we easily deduce that

$$
W_{p}^{p}\left(\pi_{k, j}^{(n)}, \pi_{u, j}\right) \leq W_{p}^{p}\left(\pi_{k, j-1}^{(n)}, \pi_{u, j-1}\right)+\int W_{p}^{p}\left(\delta_{x_{j-1}} Q_{\frac{k+j}{n}}, \delta_{y_{j-1}} Q_{u}\right) \Gamma_{u, j-1}^{(n, k)}\left(d x_{1}, d y_{1}, \ldots, d x_{j-1}, d y_{j-1}\right) .
$$

Since

$$
W_{p}\left(\delta_{x_{j-1}} Q_{\frac{k+j}{n}}, \delta_{y_{j-1}} Q_{u}\right) \leq C_{1} d\left(x_{j-1}, y_{j-1}\right)+C_{2}\left[1+d\left(y_{j-1}, x_{0}\right)\right]\left|u-\frac{k+j}{n}\right|^{K} .
$$

This leads to

$$
W_{p}\left(\pi_{k, j}^{(n)}, \pi_{u, j}\right) \leq\left(1+C_{1}\right) W_{p}\left(\pi_{k, j-1}^{(n)}, \pi_{u, j-1}\right)+C_{2}\left(1+\kappa_{2}\right)\left|u-\frac{k+j}{n}\right|^{\kappa} .
$$

The results follows by a finite induction.

Finally, note that Condition 1 of Definition 1 follows from induction and the point 2 of Proposition 3, because using the same type of arguments, we have

$$
W_{p}\left(\pi_{v, j}, \pi_{u, j}\right) \leq\left(1+C_{1}\right) W_{p}\left(\pi_{v, j-1}, \pi_{u, j-1}\right)+C_{2}\left(1+\kappa_{2}\right)|u-v|^{k} .
$$

The proof of the Theorem is now complete. 


\subsection{Mixing conditions}

We now introduce another useful coefficient: the $\tau$-mixing coefficient introduced and studied in Dedecker and Prieur (2004) that we will adapt to our triangular arrays. This coefficient has been introduced for Banach spaces $E$. In the sequel, we denote by $\Lambda_{1}(E)$ the set of 1-Lipschitz functions from $E$ to $\mathbb{R}$. Assume first that $E=\mathbb{R}$ and as for the $\beta$-mixing coefficients, set $X_{n, j}=0$ for $j>n$. Then setting

$$
U\left(\mathcal{F}_{i}^{(n)}, X_{n, i+j}\right)=\sup \left\{\left|\mathbb{E}\left[f\left(X_{n, i+j} \mid \mathcal{F}_{i}^{(n)}\right)\right]-\mathbb{E}\left[f\left(X_{n, i+j}\right)\right]\right|: \quad f \in \Lambda_{1}(\mathbb{R})\right\},
$$

the $\tau_{n}$-mixing coefficient for the sequence $\left(X_{j}^{(n)}\right)_{j \in \mathbb{Z}}$ is defined by

$$
\tau_{n}(j)=\sup _{i \in \mathbb{Z}} \mathbb{E}\left[U\left(\mathcal{F}_{i}^{(n)}, X_{n, i+j}\right)\right] .
$$

Now for a general metric space $E$, the $\tau_{n}$-mixing coefficient is defined by

$$
\tau_{n}(j)=\sup _{f \in \Delta_{1}(E)} \sup _{i \in \mathbb{Z}} \mathbb{E}\left[U\left(\mathcal{F}_{i}^{(n)}, f\left(X_{n, i+j}\right)\right)\right] .
$$

Note that, if $\widetilde{X}_{n, i}$ denotes a copy of $X_{n, i}$,

$$
\tau_{n}(j) \leq \sup _{i \in \mathbb{Z}} \mathbb{E} d\left(X_{n, i}, \widetilde{X}_{n, i}\right),
$$

For bounding this mixing coefficient, the following assumption, which strengthens assumption $\mathbf{B 2}$ in the case $m \geq 2$ and $p=1$, will be needed.

B4 There exists a positive real number $\epsilon$ such that for all $\left(u, u_{1}, \ldots, u_{m}\right) \in[0,1]^{m+1}$ satisfying $\left|u_{i}-u\right|<\epsilon$ for $1 \leq i \leq m$, we have

$$
W_{1}\left(\delta_{x} Q_{u_{1}} \cdots Q_{u_{m}}, \delta_{y} Q_{u_{1}} \cdots Q_{u_{m}}\right) \leq r d(x, y),
$$

where $m$ and $r$ are defined in assumption $\mathbf{B 2}$.

Proposition 4. Assume that assumptions $\mathbf{B 2}$ and $\mathbf{B} 4$ hold true. Then there exists $C>0$ and $\rho \in(0,1)$, only depending on $m, r, C_{1}, \epsilon$ such that

$$
\tau_{n}(j) \leq C \rho^{j}
$$

Proof of Proposition 4 We first consider the case $n \geq m / \epsilon$. Now if $k$ is an integer such that $k+m-1 \leq n$, note that assumption $\mathbf{B} 4$ entails that

$$
W_{1}\left(\mu Q_{\frac{k}{n}} \cdots Q_{\frac{k+m-1}{n}}, v Q_{\frac{k}{n}} \cdots Q_{\frac{k+m-1}{n}}\right) \leq r W_{1}(\mu, v),
$$

where the probability measures $\mu$ and $v$ have both a finite first moment. If $j=m t+s$, we get from (9) and Assumption B2,

$$
\tau_{n}(j) \leq C_{1}^{s} r^{t} \sup _{i \in \mathbb{Z}} \mathbb{E}\left[W_{1}\left(\delta_{X_{n, i}}, \pi_{i}^{(n)}\right)\right] \leq 2 \sup _{i \in \mathbb{Z}} \mathbb{E} d\left(X_{n, i}, x_{0}\right) \cdot C_{1}^{s} r^{t}
$$

We have seen in the proof of Theorem 3 that $\sup _{n \in \mathbb{Z}, i \leq n} \mathbb{E} d\left(X_{n, i}, x_{0}\right)<\infty$.

Now assume that $n<m / \epsilon$. If $j \leq n$, we have

$$
\tau_{n}(j) \leq 2 \sup _{i \in \mathbb{Z}} \mathbb{E} d\left(X_{n, i}, x_{0}\right) \cdot C_{1}^{m / \epsilon}
$$

Now if $j>n$, we have since $\left(X_{n, j}\right)_{j \leq 0}$ is stationary with transition kernel $Q_{0}$,

$$
\tau_{n}(j) \leq 2 \sup _{i \in \mathbb{Z}} \mathbb{E} d\left(X_{n, i}, x_{0}\right) \cdot C_{1}^{m / \epsilon+m} r^{\left[\frac{j-n}{m}\right]} .
$$

This leads to the result for $\rho=r^{1 / m}$ and an appropriate choice of $C>0 . \square$ 
Note. Let us remind that Proposition 4 implies a geometric decrease for the covariances. This is a consequence of the following property. If $f: E \rightarrow \mathbb{R}$ is measurable and bounded and $g: E \rightarrow \mathbb{R}$ is measurable and Lipschitz, we have

$$
\operatorname{Cov}\left(f\left(X_{n, i}\right), g\left(X_{n, i+j}\right)\right) \leq\|f\|_{\infty} \cdot \delta(g) \cdot \tau_{n}(j)
$$

\subsection{An extension to $q$-order Markov chains}

We start with an extension of our result to Markov sequences of order $q \geq 1$ and taking values in the Polish space $(E, d)$. Let $\left\{S_{u}: u \in[0,1]\right\}$ be a family of probability kernels from $\left(E^{q}, \mathcal{B}\left(E^{q}\right)\right)$ to $(E, \mathcal{B}(E))$. The two following assumptions will be used.

H1 For all $\mathbf{x} \in E^{q}, S_{u}(\mathbf{x}, \cdot) \in \mathcal{P}_{p}(E)$.

H2 There exist non-negative real numbers $a_{1}, a_{2}, \ldots, a_{q}$ satisfying $\sum_{j=1}^{q} a_{j}<1$ and such that for all $(u, \mathbf{x}, \mathbf{y}) \in$ $[0,1] \times E^{q} \times E^{q}$,

$$
W_{p}\left(S_{u}(\mathbf{x}, \cdot), S_{u}(\mathbf{y}, \cdot)\right) \leq \sum_{j=1}^{q} a_{j} d\left(x_{j}, y_{j}\right)
$$

H2 There exists a positive real number $C$ and $\kappa \in(0,1)$ such that for all $(u, v, \mathbf{x}) \in[0,1] \times[0,1] \times E^{q}$,

$$
W_{p}\left(S_{u}(\mathbf{x}, \cdot), S_{v}(\mathbf{x}, \cdot)\right) \leq C\left(1+\sum_{j=1}^{q} d\left(x_{j}, x_{0}\right)\right)|u-v|^{k} .
$$

To define Markov chains, we consider the family of Markov kernels $\left\{Q_{u}: u \in[0,1]\right\}$ on the measurable space $\left(E^{q}, \mathcal{B}(E)^{q}\right)$ and defined by

$$
Q_{u}(\mathbf{x}, d \mathbf{y})=S_{u}\left(\mathbf{x}, d y_{q}\right) \otimes \delta_{x_{2}}\left(y_{1}\right) \otimes \cdots \otimes \delta_{x_{q}}\left(d y_{q-1}\right) .
$$

Corollary 2. If the assumptions H1-H3 hold true then Theorem 3 and Proposition 4 apply.

Proof of Corollary 10 Assumption $\mathbf{H 1}$ entails B1. Then we check assumption B3. If $(u, v, \mathbf{x}) \in[0,1] \times$ $[0,1] \times E^{q}$, let $\alpha_{\mathbf{x}, u, v}$ be a coupling of the two probability distributions $S_{u}(\mathrm{x}, \cdot)$ and $S_{v}(\mathrm{x}, \cdot)$. Then

$$
\gamma_{\mathbf{x}, u, v}\left(d \mathbf{y}, d \mathbf{y}^{\prime}\right)=\alpha_{\mathbf{x}, u, v}\left(d y_{q}, d y_{q}^{\prime}\right) \otimes_{j=1}^{q-1} \delta_{x_{j+1}}\left(d y_{j}\right) \otimes \delta_{x_{j+1}}\left(d y_{j}^{\prime}\right)
$$

defines a coupling of the two measures $\delta_{\mathbf{x}} Q_{u}$ and $\delta_{\mathbf{x}} Q_{v}$. We have

$$
W_{p}\left(\delta_{\mathbf{x}} Q_{u}, \delta_{\mathbf{x}} Q_{v}\right) \leq\left[\int d\left(y_{q}, y_{q}^{\prime}\right)^{p} \alpha_{\mathbf{x}, u, v}\left(d y_{q}, d y_{q}^{\prime}\right)\right]^{1 / p} .
$$

By taking the infinimum of the last bound over all the couplings, we get

$$
W_{p}\left(\delta_{\mathbf{x}} Q_{u}, \delta_{\mathbf{x}} Q_{v}\right) \leq W_{p}\left(S_{u}(\mathbf{x}, \cdot), S_{v}(\mathbf{x}, \cdot)\right),
$$

which shows B3, using assumption $\mathbf{H 3}$.

Finally, we check assumptions $\mathbf{B} 2$ and $\mathbf{B 4}$. For an integer $m \geq 1,\left(u_{1}, \ldots, u_{m}\right) \in[0,1]^{m}$ and $(\mathbf{x}, \mathbf{y}) \in E^{q} \times E^{q}$, 
we denote by $\alpha_{\mathbf{x}, \mathbf{y}, u}$ an optimal coupling of $\left(S_{u}(\mathbf{x}, \cdot), S_{u}(\mathbf{y}, \cdot)\right)$. From Villani (2009), Corollary 5.22, there exists a measurable choice of $(\mathbf{x}, \mathbf{y}) \mapsto \alpha_{\mathbf{x y}, u}$. We define

$$
\gamma_{m, u_{1}, \ldots, u_{m}}^{\left(\mathbf{x}_{\mathbf{q}+1} \mathbf{y}_{\mathbf{q}+1}\right)}\left(d x_{q+1}, \ldots, d x_{q+m}, d y_{q+1}, \ldots, d y_{q+m}\right)=\prod_{i=q+1}^{q+m} \alpha_{\mathbf{x}_{\mathbf{i}}, \mathbf{y}_{\mathbf{i}}, u_{i}}\left(d x_{i}, d y_{i}\right),
$$

where $\mathbf{x}_{\mathbf{i}}=\left(x_{i-1}, \ldots, x_{i-q}\right)$. Let $\Omega=E^{m} \times E^{m}$ endowed with its Borel sigma field and the probability measure $\mathbb{P}=\gamma_{m, u_{1}, \ldots, u_{m}}^{\left(\mathbf{x}_{\mathbf{q}+1} \mathbf{y}_{\mathbf{q}+1}\right)}$. Then we define the random variables $Z_{j}^{\mathbf{x}_{\mathbf{q}+1}}=x_{j}, Z_{j}^{\mathbf{y}_{\mathbf{q}+1}}=y_{j}$ for $1 \leq j \leq q$ and for $1 \leq j \leq m$, $Z_{q+j}^{\mathbf{x}_{\mathbf{q}+1}}\left(\omega_{1}, \omega_{2}\right)=\omega_{1, j}, Z_{q+j}^{\mathbf{y}_{\mathbf{q}+1}}\left(\omega_{1}, \omega_{2}\right)=\omega_{2, j}$ for $j=1, \ldots, m$. By definition of our couplings, we have

$$
\mathbb{E}^{1 / p}\left[d\left(Z_{k}^{\mathbf{x}_{\mathbf{q}+\mathbf{1}}}, Z_{k}^{\mathbf{y}_{\mathbf{q}+1}}\right)^{p}\right] \leq \sum_{j=1}^{q} a_{j} \mathbb{E}^{1 / p}\left[d\left(Z_{k-j}^{\mathbf{x}_{\mathbf{q}+1}}, Z_{k-j}^{\mathbf{y}_{\mathbf{q}+1}}\right)^{p}\right] .
$$

Using a finite induction, we obtain

$$
\mathbb{E}^{1 / p}\left[d\left(Z_{k}^{\mathbf{x}_{\mathbf{q}+1}}, Z_{k}^{\mathbf{y}_{\mathbf{q}+1}}\right)^{p}\right] \leq \alpha^{\frac{k}{q}} \max _{1 \leq j \leq q} d\left(x_{j}, y_{j}\right),
$$

where $\alpha=\sum_{j=1}^{q} a_{j}$. Setting $X_{m}^{\mathbf{x}}=\left(Z_{m-q+1}^{\mathbf{x}}, \ldots, Z_{m}^{\mathbf{x}}\right)$, this entails

$$
\begin{aligned}
W_{p}\left(\delta_{\mathbf{x}} Q_{u_{1}} \cdots Q_{u_{m}}, \delta_{\mathbf{y}} Q_{u_{1}} \cdots Q_{u_{m}}\right) & \leq \mathbb{E}^{1 / p}\left[d_{q}\left(X_{m}^{\mathbf{x}}, X_{m}^{\mathbf{y}}\right)^{p}\right] \\
& \leq \sum_{j=1}^{q} \alpha^{\frac{m-j+1}{q}} \max _{1 \leq j \leq q} d\left(x_{j}, y_{j}\right) \\
& \leq \sum_{j=1}^{q} \alpha^{\frac{m-j+1}{q}} \cdot d_{q}(\mathbf{x}, \mathbf{y}) .
\end{aligned}
$$

Then B2-B4 are satisfied if $m$ is large enough by noticing that $W_{1} \leq W_{p}$.

\subsection{Examples of locally stationary Markov chains}

Natural examples of a $q$-order Markov chain satisfying the previous assumptions are based on time-varying autoregressive process. More precisely, if $E$ and $G$ are measurable spaces and $F:[0,1] \times E^{q} \times G \rightarrow E$, the triangular array $\left\{X_{n, i}: i \leq n, n \in \mathbb{Z}^{+}\right\}$is defined recursively by the equations

$$
X_{n, i}=F\left(\frac{i}{n}, X_{n, i-1}, \ldots, X_{n, i-q}, \varepsilon_{i}\right), \quad i \leq n,
$$

where the usual convention is to assume that

$$
X_{n, i}=F\left(0, X_{n, i-1}, \ldots, X_{n, i-q}, \varepsilon_{i}\right), \quad i \leq 0 .
$$

Then, if $S_{u}(\mathbf{x}, \cdot)$ denotes the distribution of $F\left(u, x_{q}, \ldots, x_{1}, \varepsilon_{1}\right)$, we have

$$
\begin{aligned}
& W_{p}\left(S_{u}(\mathbf{x}, \cdot), S_{u}(\mathbf{y}, \cdot)\right) \leq \mathbb{E}^{1 / p}\left[d\left(F\left(u, x_{q}, \ldots, x_{1}, \varepsilon_{1}\right), F\left(u, y_{q}, \ldots, y_{1}, \varepsilon_{1}\right)\right)^{p}\right], \\
& W_{p}\left(S_{u}(\mathbf{x}, \cdot), S_{v}(\mathbf{x}, \cdot)\right) \leq \mathbb{E}^{1 / p}\left[d\left(F\left(u, x_{q}, \ldots, x_{1}, \varepsilon_{1}\right), F\left(v, x_{q}, \ldots, x_{1}, \varepsilon_{1}\right)\right)^{p}\right] .
\end{aligned}
$$


Then the assumptions $\mathbf{H 1}-\mathbf{H 3}$ are satisfied if for all $(u, v, \mathbf{x}, \mathbf{y}) \in[0,1] \times[0,1] \times E^{q} \times E^{q}$,

$$
\begin{gathered}
\mathbb{E}^{1 / p}\left[d\left(F\left(u, x_{q}, \ldots, x_{1}\right), x_{0}\right)^{p}\right]<\infty, \\
\mathbb{E}^{1 / p}\left[d\left(F\left(u, x_{q}, \ldots, x_{1}\right), F\left(u, y_{q}, \ldots, y_{1}\right)\right)^{p}\right] \leq \sum_{j=1}^{q} a_{j} d\left(x_{q-j+1}, y_{q-j+1}\right)
\end{gathered}
$$

and

$$
\mathbb{E}^{1 / p}\left[d\left(F\left(u, x_{q}, \ldots, x_{1}\right), F\left(v, x_{q}, \ldots, x_{1}\right)\right)^{p}\right] \leq C\left(1+\sum_{j=1}^{q} d\left(x_{j}, x_{0}\right)\right) \cdot|u-v|^{\kappa} .
$$

A typical example of such time-varying autoregressive process is the univariate tv-ARCH process for which

$$
X_{n, i}=\xi_{i} \sqrt{a_{0}(i / n)+\sum_{j=1}^{q} a_{j}(i / n) X_{n, i-j}^{2}},
$$

with $\mathbb{E} \xi_{t}=0$, Var $\xi_{t}=1$. The previous assumptions are satisfied for the square of this process if the $a_{j}$ 's are $\kappa-$ Hölder continuous and if

$$
\left\|\xi_{t}^{2}\right\|_{p} \cdot \sup _{u \in[0,1]} \sum_{j=1}^{q} a_{j}(u)<1 \text {, for some } p \geq 1 .
$$

See Fryzlewicz et al. (2008) and Truquet (2016) for the use of those processes for modeling financial data.

Note. The approximation of time-varying autoregressive processes by stationnary processes is discussed in several papers. See for instance Subba Rao (2006) for linear autoregressions with time varying random coefficients, Vogt (2012) for nonlinear time-varying autoregressions or Zhang and Wu (2015) for additional results in the same setting. However, the approximating stationary process of (10) is given by

$$
X_{i}(u)=F\left(u, X_{i-1}(u), \ldots, X_{i-q}(u), \varepsilon_{i}\right) .
$$

Note that $W_{p}\left(\pi_{k}^{(n)}, \pi_{u}\right) \leq \mathbb{E}^{1 / p}\left[d\left(X_{k}^{(n)}, X_{k}(u)\right)^{p}\right]$ and the aforementioned references usually study a control of this upper bound by $\left|u-\frac{k}{n}\right|^{K}+\frac{1}{n^{\kappa}}$. Note that in the case of autoregressive processes, a coupling of the time-varying processes and its stationary approximation is already defined because the same noise process is used in both cases. However it is possible to construct some examples for which $\pi_{k}^{(n)}=\pi_{u}$ and $\mathbb{E}^{1 / p}\left[d\left(X_{n, k}, X_{k}(u)\right)^{p}\right] \neq 0$, i.e the coupling used is not optimal. Nevertheless, it is still possible to obtain an upper bound of $\mathbb{E}^{1 / p}\left[d\left(X_{k}^{(n)}, X_{k}(u)\right)^{p}\right]$ using our results. To this end, let us assume that $q=1$ (otherwise one can use vectors of $q$-successive coordinates to obtain a Markov chain of order 1) if $u \in[0,1]$, and we consider the Markov kernel form $\left(E^{2}, \mathcal{B}\left(E^{2}\right)\right)$ to itself, given by

$$
Q_{v}^{(u)}\left(x_{1}, x_{2}, A\right)=\mathbb{P}\left(\left(F\left(v, x_{1}, \varepsilon_{1}\right), F\left(u, x_{2}, \varepsilon_{1}\right)\right) \in A\right), \quad A \in \mathcal{B}\left(E^{2}\right), \quad v \in[0,1] .
$$

One can show that the family $\left\{Q_{v}^{(u)}: v \in[0,1]\right\}$ satisfies the assumptions B1 - B4 for the metric

$$
d_{2}\left[\left(x_{1}, x_{2}\right),\left(y_{1}, y_{2}\right)\right]=\left(d\left(x_{1}, y_{1}\right)^{p}+d\left(x_{2}, y_{2}\right)^{p}\right)^{1 / p} .
$$

Moreover, the constant in Theorem 3 does not depend on $u \in[0,1]$. Then Lemma 4 guarantees that there exists a positive constant $C$ not depending on $k, n, u$ such that

$$
\mathbb{E}^{1 / p}\left(d\left(X_{k, n}, X_{k}(u)\right)^{p}\right) \leq C\left[\left|u-\frac{k}{n}\right|^{\kappa}+\frac{1}{n^{k}}\right] .
$$


Iteration of random affine functions Here we assume that for each $u \in[0,1]$, there exists a sequence $\left(A_{t}(u), B_{t}(u)\right)_{t \in \mathbb{Z}}$ of i.i.d random variables such that $A_{t}(u)$ takes its values in the space $\mathcal{M}_{d}$ of squares matrices of dimension $d$ with real coefficients and $B_{t}(u)$ takes its values in $E=\mathbb{R}^{d}$. Let $\|\cdot\|$ a norm on $E$. We also denote by $\|\cdot\|$ the corresponding operator norm on $\mathcal{M}_{d}$. We then consider the following recursive equations

$$
X_{n, i}=A_{i}\left(\frac{i}{n}\right) X_{n, i-1}+B_{i}\left(\frac{i}{n}\right) .
$$

Local approximation of these autoregressive processes by their stationary versions $X_{t}(u)=A_{t}(u) X_{t-1}(u)+$ $B_{t}(u)$ is studied is studied by Subba Rao (2006). In this subsection, we will derive similar results using our Markov chain approach. For each $u \in[0,1]$, we denote by $\gamma_{u}$ the top Lyapunov exponent of the sequence $\left(A_{t}(u)\right)_{t \in \mathbb{Z}}$, i.e

$$
\gamma_{u}=\inf _{n \geq 1} \frac{1}{n} \mathbb{E} \log \left\|A_{n}(u) A_{n-1}(u) \cdots A_{1}(u)\right\| .
$$

We assume that there exists $t \in(0,1)$ such that

R1 for all $u \in[0,1], \mathbb{E}\left\|A_{1}(u)\right\|^{t}<\infty, \mathbb{E}\left\|B_{1}(u)\right\|^{t}<\infty$ and $\gamma_{u}<0$.

$\mathbf{R 2}$ There exists $C>0$ and $\widetilde{\kappa} \in(0,1)$ such that for all $(u, v) \in[0,1]^{2}$,

$$
\mathbb{E}\left\|A_{1}(u)-A_{1}(v)\right\|^{t}+\mathbb{E}\left\|B_{1}(u)-B_{1}(v)\right\|^{t} \leq C|u-v|^{\widetilde{\kappa}} .
$$

Proposition 5. For $s \in(0,1)$, we set $d(x, y)=\|x-y\|^{s}$. Assume that assumptions $\mathbf{R} 1-\mathbf{R 2}$ hold true. Then there exists $s \in(0, t)$ such that Theorem 3 applies with $p=1, \kappa=\widetilde{\tau \kappa}$ and $d(x, y)=\|x-y\|^{s}$ and $x_{0}=0$.

\section{Notes}

1. Using the remark in the Note of Section 3.3, we also have $\mathbb{E}\left\|X_{n, k}-X_{k}(u)\right\|^{s} \leq C\left(\left|u-\frac{k}{n}\right|^{\widetilde{K}}+\frac{1}{n^{k}}\right)^{s}$, where the process $\left(X_{j}(u)\right)_{j \in \mathbb{Z}}$ satisfies the iterations $X_{k}(u)=A_{k}(u) X_{k-1}(u)+B_{k}(u)$. Then the triangular array $\left\{X_{n, k}: k \leq n, n \in \mathbb{Z}^{+}\right\}$is locally stationary in the sense given in Vogt (2012) (see Definition 2.1 of that paper).

2. One can also give additional results for the Wasserstein metric of order $p \geq 1$ and $d(x, y)=\|x-y\|$ if

$$
\mathbb{E}\left\|A_{1}(u)\right\|^{p}+\mathbb{E}\left\|B_{1}(u)\right\|^{p}<\infty, \quad \mathbb{E}^{1 / p}\left\|A_{1}(u)-A_{1}(v)\right\|^{p}+\mathbb{E}^{1 / p}\left\|B_{1}(u)-B_{1}(v)\right\|^{p} \leq C|u-v|^{K}
$$

and there exists an integer $m \geq 1$ such that $\sup _{u \in[0,1]} \mathbb{E}\left\|A_{m}(u) \cdots A_{1}(u)\right\|^{p}<1$. In particular, one can recover results about the local approximation of $\mathrm{tv}$-AR processes defined by

$$
X_{n, i}=\sum_{j=1}^{q} a_{j}(i / n) X_{n, i-j}+\sigma(i / n) \varepsilon_{i}
$$

by vectorizing $q$ successive coordinates and assuming $\kappa-$ Hölder continuity for the $a_{j}$ 's and $\sigma$. Details are omitted. 
Proof of Proposition 5 For all $(x, u) \in \mathbb{R}^{d} \times[0,1]$, the measure $\delta_{x} Q_{u}$ is the probability distribution of the random variable $A_{k}(u) x+B_{k}(u)$. Condition $\mathbf{A} \mathbf{1}$ of Theorem 3 follows directly from assumption $\mathbf{R} \mathbf{1}$ (whatever the value of $s \in(0, t))$. Moreover, we have for $s \in(0, t)$,

$$
\begin{aligned}
W_{1}\left(\delta_{x} Q_{u}, \delta_{x} Q_{v}\right) & \leq \mathbb{E}\left\|A_{k}(u)-A_{k}(v)\right\|^{s} \cdot\|x\|^{s}+\mathbb{E}\left\|B_{k}(u)-B_{k}(v)\right\|^{s} \\
& \leq\left(1+\|x\|^{s}\right) \cdot\left(\mathbb{E}^{\frac{s}{t}}\left\|A_{k}(u)-A_{k}(v)\right\|^{t}+\mathbb{E}^{\frac{s}{t}}\left\|B_{k}(u)-B_{k}(v)\right\|^{t}\right) .
\end{aligned}
$$

This entails condition A3, using assumption R2. Next, if $u \in[0,1]$, the conditions $\gamma_{u}<0$ and $\mathbb{E}\left\|A_{t}(u)\right\|^{t}<\infty$ entail the existence of an integer $k_{u}$ and $s_{u} \in(0, t)$ such that $\mathbb{E}\left\|A_{k_{u}}(u) A_{k_{u}-1}(u) \cdots A_{1}(u)\right\|^{s_{u}}<1$ (see for instance Francq and Zakoïan (2010), Lemma 2.3). Using the axiom of choice, let us select for each $u$, a couple $\left(k_{u}, s_{u}\right)$ satisfying the previous property. From assumption R2, the set

$$
O_{u}=\left\{v \in[0,1]: \mathbb{E}\left\|A_{k_{u}}(v) A_{k_{u}-1}(v) \cdots A_{1}(v)\right\|^{s_{u}}<1\right\}
$$

is an open set of $[0,1]$. By a compactness argument, there exist $u_{1}, \ldots, u_{d} \in[0,1]$ such that $[0,1]=\cup_{i=1}^{d} O_{u_{i}}$. Then setting $s=\min _{1 \leq i \leq d} s_{u_{i}}$ and denoting by $m$ the lowest common multiple of the integers $k_{u_{1}}, \ldots, k_{u_{d}}$, we have from assumption $\mathbf{R} 2$,

$$
r=\sup _{u \in[0,1]} \mathbb{E}\left\|A_{m}(u) \cdots A_{1}(u)\right\|^{s}<1 .
$$

This entails condition $\mathbf{B} 2$ for this choice of $s, m$ and $r$. Indeed, we have

$$
W_{1}\left(\delta_{x} Q_{u}^{m}, \delta_{y} Q_{u}^{m}\right) \leq \mathbb{E}\left\|A_{m}(u) \cdots A_{1}(u)(x-y)\right\|^{s} \leq r d(x, y) .
$$

Note also that condition $\mathbf{B} 4$ easily follows from the uniform continuity of the application $\left(u_{1}, \ldots, u_{m}\right) \mapsto$ $\mathbb{E}\left\|A_{m}\left(u_{1}\right) \cdots A_{1}\left(u_{m}\right)\right\|^{s}$.ロ.

Time-varying integer-valued autoregressive processes (tv-INAR) Stationary INAR processes are widely used in the time series community. This time series model has been proposed by Al Osh and Alzaid (1987) and a generalization to several lags was studied in Jin-Guan and Yuan (1991). In this paper, we introduce a locally stationary version of such processes. For $u \in[0,1]$ and $j=1,2, \ldots, q+1$, we consider a probability $\zeta_{j}(u)$ on the nonnegative integers and for $1 \leq j \leq q$, we denote by $\alpha_{j}(u)$ the mean of the distribution $\zeta_{j}(u)$. Now let

$$
X_{n, k}=\sum_{j=1}^{q} \sum_{i=1}^{X_{n, k-j}} Y_{j, i}^{(n, k)}+\eta_{k}^{(n)}
$$

where for each integer $n \geq 1$, the family $\left\{Y_{j, i}^{(n, k)}, \eta_{h}^{(n)}:(k, j, i, h) \in \mathbb{Z}^{4}\right\}$ contains independent random variables and such that for $1 \leq j \leq q, Y_{j, i}^{(n, k)}$ has probability distribution $\zeta_{j}(k / n)$ and $\eta_{k}^{(n)}$ has probability distribution $\zeta_{q+1}(k / n)$. Note that, one can define a corresponding stationary autoregressive process. To this end, we denote by $F_{j, u}$ the cumulative distribution of the probability $\zeta_{j}(u)$ and we consider a family $\left\{U_{j, i}^{(k)}, V_{k}:(i, j, k) \in \mathbb{Z}^{3}\right\}$ of i.i.d random variables uniformly distributed over $[0,1]$ and we set $Y_{j, i}^{(n, k)}=$ $F_{j, k / n}^{-1}\left(U_{j, i}^{(k)}\right)$ where for a cumulative distribution function $G, G^{-1}$ denotes its left continuous inverse. Then one can consider the stationary version

$$
X_{k}(u)=\sum_{j=1}^{q} \sum_{i=1}^{X_{k-j}(u)} F_{j, u}^{-1}\left(U_{j, i}^{(k)}\right)+F_{q+1, u}^{-1}\left(V_{k}\right) .
$$

The following result is a consequence of Corollary 10. Only the case $p=1$ is considered here. 
Corollary 3. Let $d$ be the usual distance on $\mathbb{R}$. Assume that $\sup _{u \in[0,1]} \sum_{j=1}^{q} \alpha_{j}(u)<1, \zeta_{q+1}$ has a finite first moment and $W_{1}\left(\zeta_{j}(u), \zeta_{j}(v)\right) \leq C|u-v|^{\kappa}$ for $C>0, \kappa \in(0,1)$ and $1 \leq j \leq q+1$. Then Theorem 3 and Proposition 4 apply.

Example. Stationary INAR processes are often used when $\zeta_{j}$ is a Bernoulli distribution of parameter $\alpha_{j} \in(0,1)$ for $1 \leq j \leq q$ and $\zeta_{q+1}$ is a Poisson distribution of parameter $\lambda$. This property guarantees that the marginal distribution is also Poissonian. Condition $\sum_{j=1}^{q} \alpha_{j}<1$ is a classical condition ensuring the existence of a stationary solution for this model. In the locally stationnary case, let $U$ be a random variable following a uniform distribution over $[0,1]$ and $\left(N_{t}\right)_{t}$ be a Poisson process of intensity 1 . Then, we have

$$
\begin{gathered}
W_{1}\left(\zeta_{j}(u), \zeta_{j}(v)\right) \leq \mathbb{E}\left|\mathbb{1}_{U \leq \alpha_{j}(u)}-\mathbb{1}_{U \leq \alpha_{j}(v)}\right| \leq\left|\alpha_{j}(u)-\alpha_{j}(v)\right|, \\
W_{1}\left(\zeta_{q+1}(u), \zeta_{q+1}(v)\right) \leq \mathbb{E}\left|N_{\lambda(u)}-N_{\lambda(v)}\right| \leq|\lambda(u)-\lambda(v)| .
\end{gathered}
$$

Then the assumptions of Corollary 3 are satisfied if the functions $\alpha_{j}$ and $\lambda$ are $\kappa$-Hölder continuous and if $\sum_{j=1}^{q} \alpha_{j}(u)<1, u \in[0,1]$.

Note. One can also state a result for $p \geq 1$. This case is important if we have to compare the expectation of some polynomials of the time-varying process with its the stationary version. However, in the example given above, a naive application of our results will require $\sum_{j=1}^{q} \alpha_{j}(u)^{1 / p}<1$. Moreover, one can show that a $\kappa-$ Hölder regularity on $\alpha_{j}$ and $\lambda$ entails a $\frac{\kappa}{p}-$ Hölder regularity in Wasserstein metrics. For instance if $q=1$, we have $W_{p}\left(\delta_{0} Q_{u}, \delta_{0} Q_{v}\right) \geq|\lambda(u)-\lambda(v)|^{1 / p}$. In order to avoid these unnatural conditions for this model, we will use the approach developed in Section 4.

\section{Local stationarity and drift conditions}

In this section, we will use some drift and minoration conditions to extend the Dobrushin's contraction technique of Section 2. A key result for this section is Lemma 5 which is adapted from Lemma 6.29 in Douk et al. (2014). This result gives sufficient conditions for contracting Markov kernels with respect to norm induced by a particular Foster-Lyapunov drift function. The original argument for such contraction properties is due to Hairer and Mattingly (2011). This important result will enable us to consider additional examples of locally stationary Markov chains with non compact state spaces. For a function $V: E \rightarrow[1, \infty)$, we define the $V$-norm of signed measure $\mu$ on $(E, \mathcal{B}(E))$ by

$$
\|\mu\|_{V}=\sup _{f:|f(x)| \leq V(x)}\left|\int f d \mu\right| .
$$

\subsection{General result}

We will assume that there exists a measurable function $V: E \rightarrow[1, \infty)$ such that

F1 there exist $\epsilon>0, \lambda \in(0,1)$, an integer $m \geq 1$ and two real numbers $b>0, K \geq 1$ such that for all $u, u_{1}, \ldots, u_{m} \in[0,1]$ satisfying $\left|u-u_{i}\right| \leq \epsilon$,

$$
Q_{u} V \leq K V, \quad Q_{u_{1}} \cdots Q_{u_{m}} V \leq \lambda V+b .
$$

Moreover, there exists $\eta>0, R>2 b /(1-\lambda)$ and a probability measure $v \in \mathcal{P}(E)$ such that

$$
\delta_{x} Q_{u_{1}} \cdots Q_{u_{m}} \geq \eta \nu, \text { if } V(x) \leq R
$$


F2 there exist $\kappa \in(0,1)$ and a function $\widetilde{V}: E \rightarrow(0, \infty)$ such that $\sup _{u \in[0,1]} \pi_{u} \widetilde{V}<\infty$ and for all $x \in E$, $\left\|\delta_{x} Q_{u}-\delta_{x} Q_{v}\right\|_{V} \leq \widetilde{V}(x)|u-v|^{\kappa}$.

We first give some properties of the Markov kernels $Q_{u}$ with respect to the $V$-norm.

Proposition 6. Assume that assumptions F1 - F2 hold true.

1. There exist $C>0$ and $\rho \in(0,1)$ such that for all $x \in E$,

$$
\sup _{u \in[0,1]}\left\|\delta_{x} Q_{u}^{j}-\pi_{u}\right\|_{V} \leq C V(x) \rho^{j} .
$$

Moreover $\sup _{u \in[0,1]} \pi_{u} V<\infty$.

2. There exists $C>0$ such that for all $(u, v) \in[0,1]^{2}$,

$$
\left\|\pi_{u}-\pi_{v}\right\|_{V} \leq C|u-v|^{K}
$$

\section{Proof of Proposition 6.}

1. According to Lemma 5, there exists $(\gamma, \delta) \in(0,1)^{2}$ only depending $\lambda, b, \eta$, such that

$$
\Delta_{V_{\delta}}\left(Q_{u}^{m}\right)=\sup \left\{\frac{\|\mu R-v R\|_{V_{\delta}}}{\|\mu-v\|_{V_{\delta}}}: \quad \mu, v \in \mathcal{P}(E), \quad \mu V_{\delta}<\infty, \nu V_{\delta}<\infty\right\} \leq \gamma,
$$

with $V_{\delta}=1-\delta+\delta V$. From Theorem 6.19 in Douk et al. (2014) and Assumption F1, we have a unique invariant probability for $Q_{u}$, satisfying $\pi_{u} V<\infty$ and for $\mu \in \mathcal{P}(E)$ such that $\mu V<\infty$, we have

$$
\left\|\mu Q_{u}^{j}-\pi_{u}\right\|_{V_{\delta}} \leq \max _{0 \leq s \leq m-1} \Delta_{V_{\delta}}\left(Q_{u}^{s}\right) \gamma^{[j / m]}\left\|\mu-\pi_{u}\right\|_{V_{\delta}} .
$$

Note that $\|\cdot\|_{V_{\delta}} \leq\|\cdot\|_{V} \leq \frac{1}{\delta}\|\cdot\|_{V_{\delta}}$ and the two norms are equivalent. Using Lemma 6.18 in Douk et al. (2014), we have

$$
\Delta_{V_{\delta}}\left(Q_{u}^{s}\right)=\sup _{x \neq y} \frac{\left\|\delta_{x} Q_{u}^{s}-\delta_{y} Q_{u}^{s}\right\|_{V_{\delta}}}{V_{\delta}(x)+V_{\delta}(y)} \leq \frac{K^{s}}{\delta} .
$$

Then it remains to show that $\sup _{u \in[0,1]} \pi_{u} V<\infty$ or equivalently $\sup _{u \in[0,1]} \pi_{u} V_{\delta}<\infty$. But this a consequence of the contraction property of the application $\mu \mapsto \mu Q_{u}^{m}$ on the space

$$
\mathcal{M}_{\delta}=\left\{\mu \in \mathcal{P}(E): \mu V_{\delta}<\infty\right\}
$$

endowed with the distance $d_{\delta}(\mu, v)=\|\mu-v\|_{V_{\delta}}$, which is a complete metric space (see Proposition 6.16 in Douk et al. (2014)). Hence we have

$$
\mu-\pi_{u}=\sum_{j=0}^{\infty}\left[\mu Q_{u}^{m j}-\mu Q_{u}^{m(j+1)}\right]
$$

which defines a normally convergent series in $\mathcal{M}_{\delta}$ and

$$
\left\|\mu-\pi_{u}\right\|_{V_{\delta}} \leq \sum_{j=0}^{\infty} \gamma^{j}\left\|\mu-\mu Q_{u}^{m}\right\|_{V_{\delta}} \leq \frac{\mu V+K^{m} \mu V}{1-\gamma} .
$$

This shows that $\sup _{u \in[0,1]} \pi_{u} V<\infty$ and the proof of the first point is now complete. 
2. To prove the second point, we decompose as in the two previous sections $\pi_{u}-\pi_{v}=\pi_{u} Q_{u}^{m}-\pi_{v} Q_{u}^{m}+$ $\pi_{v} Q_{u}^{m}-\pi_{v} Q_{v}^{m}$. This leads to the inequality

$$
\left\|\pi_{u}-\pi_{v}\right\|_{V_{\delta}} \leq \frac{\left\|\pi_{v} Q_{u}^{m}-\pi_{v} Q_{v}^{m}\right\|_{V_{\delta}}}{1-\gamma}
$$

Moreover, we have

$$
\begin{aligned}
\left\|\pi_{v} Q_{u}^{m}-\pi_{v} Q_{v}^{m}\right\|_{V_{\delta}} & \leq\left\|\pi_{v} Q_{u}^{m}-\pi_{v} Q_{v}^{m}\right\|_{V} \\
& \leq \sum_{j=0}^{m-1}\left\|\pi_{v} Q_{v}^{m-j-1}\left(Q_{v}-Q_{u}\right) Q_{u}^{j}\right\|_{V} \\
& \leq \sum_{j=0}^{m-1} K^{j}\left\|\pi_{v} Q_{v}^{m-j-1}\left(Q_{v}-Q_{u}\right)\right\|_{V} \\
& \leq \sum_{j=0}^{m-1} K^{j} \cdot \pi_{v} \widetilde{V} \cdot|u-v|^{\kappa} .
\end{aligned}
$$

Hence the result follows with $C=\frac{\sup _{u \in[0,1]} \pi_{u} \widetilde{V}}{1-\gamma} \sum_{j=0}^{m-1} K^{j}$.

Now, we give our result about local stationarity.

Theorem 4. 1. Under the assumptions $\mathbf{F 1 - F 2 , ~ t h e r e ~ e x i s t s ~ a ~ p o s i t i v e ~ r e a l ~ n u m b e r ~} C$, only depending on $m, \lambda, b, K$ and $\sup _{u \in[0,1]} \pi_{u} \widetilde{V}$ such that

$$
\left\|\pi_{k}^{(n)}-\pi_{u}\right\|_{V} \leq C\left[\left|u-\frac{k}{n}\right|^{K}+\frac{1}{n^{K}}\right] .
$$

2. Assume that assumptions $\mathbf{F 1}-\mathbf{F 3}$ hold true and that in addition, for all $(u, v) \in[0,1]^{2}$,

$$
\left\|\delta_{x} Q_{u}-\delta_{x} Q_{v}\right\|_{T V} \leq L(x)|u-v|^{\kappa} \text { with } G=\sup _{\substack{u \in[0,1] \\ 1 \leq \ell^{\prime} \leq \ell}} \mathbb{E} L\left(X_{\ell}(u)\right) V\left(X_{\ell^{\prime}}(u)<\infty .\right.
$$

On $\mathcal{P}\left(E^{j}\right)$, we define the $V-$ norm by

$$
\|f\|_{V}=\sup \left\{\int f d \mu: \quad\left|f\left(x_{1}, \ldots, x_{j}\right)\right| \leq V\left(x_{1}\right)+\cdots+V\left(x_{j}\right)\right\} .
$$

Then there exists $C_{j}>0$, not depending on $k, n, u$ and such that,

$$
\left\|\pi_{k, j}^{(n)}-\pi_{u, j}\right\|_{V} \leq C_{j}\left[\left|u-\frac{k}{n}\right|^{K}+\frac{1}{n^{K}}\right] .
$$

Moreover, the triangular array of Markov chains $\left\{X_{n, k}: n \in \mathbb{Z}^{+}, k \leq n\right\}$ is locally stationary. 
Proof of Theorem 4. We assume that $n \geq \frac{m}{\epsilon}$.

1. We start with the case $j=1$. Under the assumptions of the theorem, Lemma 5 guarantees the existence of $(\gamma, \delta) \in(0,1)^{2}$ such that for all $k \leq n, \Delta_{V_{\delta}}\left(Q_{\frac{k-m+1}{n}} \cdots Q_{\frac{k}{n}}\right) \leq \gamma$ with $V_{\delta}=1-\delta+\delta V$. In the sequel, we set $Q_{k, m}=Q_{\frac{k-m+1}{n}} \cdots Q_{\frac{k}{n}}$. Then we get

$$
\left\|\pi_{k}^{(n)}-\pi_{u}\right\|_{V_{\delta}} \leq \gamma\left\|\pi_{k-m}^{(n)}-\pi_{u}\right\|_{V_{\delta}}+\left\|\pi_{u} Q_{k, m}-\pi_{u} P_{u}^{m}\right\|_{V_{\delta}} .
$$

Now using F1 and F3, we get

$$
\begin{aligned}
\left\|\pi_{u} Q_{k, m}-\pi_{u} Q_{u}^{m}\right\|_{V} & \leq \sum_{j=0}^{m-1}\left\|\pi_{u} Q_{u}^{m-j-1}\left[Q_{\frac{k-j}{n}}-Q_{u}\right] Q_{\frac{k-j+1}{n}} \cdots Q_{\frac{k}{n}}\right\|_{V} \\
& \leq \sum_{j=0}^{m-1} K^{j} \cdot \pi_{u} Q_{u}^{m-j+1} \widetilde{V} \cdot\left|u-\frac{k-j}{n}\right|^{K} \\
& \leq K^{m} \sup _{u \in[0,1]} \pi_{u} \widetilde{V} \sum_{s=k-m+1}^{k}\left|u-\frac{s}{n}\right|^{K} .
\end{aligned}
$$

This shows the result in this case, by noticing that $\|\cdot\|_{V_{\delta}} \leq\|\cdot\|_{V} \leq \delta^{-1}\|\cdot\|_{V_{\delta}}$.

Now, if $n<m / \epsilon$, we have

$$
\left\|\pi_{k}^{n}-\pi_{u}\right\|_{V} \leq \pi_{k}^{(n)} V+\sup _{u \in[0,1]} \pi_{u} V \leq K^{\frac{m}{\epsilon}}\left(1+\sup _{u \in[0,1]} \pi_{u} V\right) \leq K^{\frac{m}{\epsilon}}\left(1+\sup _{u \in[0,1]} \pi_{u} V\right) \frac{m^{\kappa}}{\epsilon^{K}} n^{-\kappa},
$$

which leads to the result.

2. Assume that the result is true for an integer $j \geq 1$. Let $f: E^{j+1} \rightarrow \mathbb{R}_{+}$be such that $f\left(x_{1}, \ldots, x_{j+1}\right) \leq$ $V\left(x_{1}\right)+\cdots+V\left(x_{j+1}\right)$. Setting $s_{j}=\sum_{i=1}^{j} V\left(x_{i}\right)$ and $g_{j}\left(x_{j+1}\right)=f\left(x_{1}, \ldots, x_{j+1}\right)$, we use the decomposition

$$
g_{j}=g_{j} \mathbb{1}_{g_{j} \leq V}+\left(g_{j}-V\right) \mathbb{1}_{V<g_{j} \leq V+s_{j}}+V \mathbb{1}_{V<g_{j} \leq V+s_{j}},
$$

and we get

$$
\begin{aligned}
\left|\delta_{x_{j+1}} Q_{\frac{k+j+1}{n}} g_{j}-\delta_{x_{j+1}} Q_{u} g_{j}\right| & \leq\left\|\delta_{x_{j+1}} Q_{\frac{k+j+1}{n}}-\delta_{x_{j+1}} Q_{u}\right\|_{V}+s_{j}\left\|\delta_{x_{j+1}} Q_{\frac{k+j+1}{n}}-\delta_{x_{j+1}} Q_{u}\right\|_{T V} \\
& \leq\left[\widetilde{V}\left(x_{j+1}\right)+\left(V\left(x_{1}\right)+\cdots+V\left(x_{j}\right)\right) L\left(x_{j}\right)\right]\left|u-\frac{k+j+1}{n}\right|^{K} .
\end{aligned}
$$

This yields to

$$
\left\|\pi_{u, j} \otimes Q_{\frac{k+j+1}{n}}-\pi_{u, j+1}\right\|_{V} \leq 2\left(\sup _{u \in[0,1]} \pi_{u} \widetilde{V}+j G\right)\left|u-\frac{k+j+1}{n}\right|^{K} .
$$

On the other hand

$$
\left\|\pi_{u, j} \otimes Q_{\frac{k+j+1}{n}}-\pi_{k, j+1}^{(n)}\right\|_{V} \leq(1+K)\left\|\pi_{k, j}^{(n)}-\pi_{u, j}\right\|_{V} .
$$

The two last bounds lead to the result using finite induction. Moreover, using the same type of arguments, one can check the continuity condition 1 of Definition 1 . 
One can also define a useful upper bound of the usual $\beta$-mixing coefficient which is useful to control covariances of unbounded functionals of the Markov chain. More precisely, we set

$$
\beta_{n}^{(V)}(j)=\sup _{k \leq n} \mathbb{E}\left\|\pi_{k}^{(n)}-\delta_{X_{n, k-j}} Q_{\frac{k-j+1}{n}} \cdots Q_{\frac{k}{n}}\right\|_{V}
$$

We have the following result which proof is straigthforward.

Proposition 7. Assume that assumption $\mathbf{F 1}$ holds true and that $n \geq m / \epsilon$. Then if $j=m g+s$, we have $\beta_{n}^{(V)}(j) \leq 2 \delta^{-1} \sup _{k \leq n} \pi_{k}^{(n)} V \cdot K^{s} \gamma^{g}$, where $\delta, \gamma \in(0,1)$ are given in Lemma 5 .

\section{Notes}

1. From the drift condition in F1, we have $\pi_{k}^{(n)} V \leq \frac{b}{1-\lambda}$ for $n \geq m / \epsilon$. Hence $\sup _{n \in \mathbb{Z}^{+}, k \leq n} \pi_{k}^{(n)} V<\infty$.

2. We did not adapt the notion of $V$-mixing given in Meyn and Tweedie (2009), Chapter 16. However, let us mention that if $A=\sup _{k \leq n, n \in \mathbb{Z}^{+}} \pi_{k}^{(n)}[|g| V]<\infty$, we get the following covariance inequality

$$
\left|\operatorname{Cov}\left(g\left(X_{n, k-j}\right), f\left(X_{n, k}\right)\right)\right| \leq 2 \delta^{-1} A K^{s} \gamma^{g},
$$

when $j=m g+s$.

\subsection{Example 1: the random walk on the nonnegative integers}

Let $p, q, r:[0,1] \rightarrow(0,1)$ three $\kappa-$ Hölder continuous functions such that $p(u)+q(u)+r(u)=1$ and $\frac{p(u)}{q(u)}<1$. For $x \in \mathbb{N}^{*}$, we set $Q_{u}(x, x)=r(u), Q_{u}(x, x+1)=p(u)$ and $Q_{u}(x, x-1)=q(u)$. Finally $Q_{u}(0,1)=1-Q_{u}(0,0)=p(u)$. In the homogeneous case, geometric ergodicity holds under the condition $p<q$. See Meyn and Tweedie (2009), Chapter 15. In this case the function $V$ defined by $V(x)=z^{x}$ is a Foster-Lyapunov function if $1<z<q / p$. For the non-homogeneous case, let $z \in(1, e)$ where $e=$ $\min _{u \in[0,1]} q(u) / p(u)$. We set $\gamma=\max _{u \in[0,1]}\left\{r(u)+p(u) z+q(u) z^{-1}\right\}$ and $\bar{p}=\max _{u \in[0,1]} p(u)$. Note that

$$
\gamma \leq 1+\bar{p}(z-1) \max _{u \in[0,1]}\left[1-\frac{q(u)}{p(u) z}\right] \leq 1+\bar{p}(z-1)\left[1-\frac{e}{z}\right]<1 .
$$

Then we have $Q_{u} V(x) \leq \gamma V(x)$ for all $x>0$ and $Q_{u} V(0)=p(u) z+(1-p(u)) \leq c=\bar{p}(z-1)+1$. For an integer $m \geq 1$, we have $Q_{u_{1}} \cdots Q_{u_{m}} V \leq \gamma^{m} V+\frac{c}{1-\gamma}$. If $m$ is large enough, we have $\frac{2 c}{(1-\gamma)\left(1-\gamma^{m}\right) V(m)}<1$. Moreover, for such $m$, if $R=V(m)$, we have $\{V \leq R\}=\{0,1, \ldots, m\}$ and if $x=0, \ldots, m$, we have $\delta_{x} Q_{u_{1}} \cdots Q_{u_{m}} \geq \eta \delta_{0}$ for a $\eta>0$. Assumption F3 is immediate. Moreover the additional condition in the second point of Theorem 4 is automatically checked with a constant function $L$.

However this example is more illustrative. Indeed parameters $p(u)$ and $q(u)$ can be directly estimated by

$$
\hat{p}(u)=\sum_{i=1}^{n-1} e_{i}(u) \mathbb{1}_{X_{n, i+1}-X_{n, i}=1}, \quad \hat{q}(u)=\sum_{i=1}^{n-1} e_{i}(u) \mathbb{1}_{X_{n, i+1}-X_{n, i}=-1},
$$

where the weights $e_{i}(u)$ are defined as in Subsection 2.3. The indicators are independent Bernoulli random variables with parameter $p\left(\frac{i+1}{n}\right)$ or $q\left(\frac{i+1}{n}\right)$ and the asymptotic behavior of the estimates is straightforward. 


\subsection{Example 2: INAR processes}

We again consider INAR processes. For simplicity, we only consider the case $q=1$ with Bernoulli counting sequences and a Poissonian noise. The parameters $\alpha(u)$ (resp. $\lambda(u)$ ) of the counting sequence (resp. the Poissonian noise) are assumed to be $\kappa-$ Hölder continuous. We will show that our results apply with drift functions $V_{p}(x)=x^{p}+1$ for an arbitrary integer $p \geq 1$. To this end, we consider a sequence $\left(Y_{i}(u)\right)_{i \geq 0}$ of i.i.d random variables following the Bernoulli distribution of parameter $\alpha(u)$ and a random variable $\xi(u)$ following the Poisson distribution of parameter $\lambda(u)$. We assume that $\xi(u)$ and the sequence $\left(Y_{i}(u)\right)_{i \geq 0}$ are independent. For $u \in[0,1]$, we have

$$
\begin{aligned}
\delta_{x} Q_{u} V_{p} & =1+\mathbb{E}\left(\alpha(u) x+\sum_{i=1}^{x}\left(Y_{i}(u)-\alpha(u)\right)+\xi(u)\right)^{p} \\
& =1+\alpha(u)^{p} x^{p}+\sum_{j=1}^{p}\left(\begin{array}{c}
p \\
j
\end{array}\right) \alpha(u)^{p-j} x^{p-j} \mathbb{E}\left(\sum_{i=1}^{x}\left(Y_{i}(u)-\alpha(u)\right)+\xi(u)\right)^{j}
\end{aligned}
$$

Using the Burkhölder inequality for martingales, we have for an integer $\ell \geq 2$,

$$
\mathbb{E}\left(\sum_{i=1}^{x}\left(Y_{i}(u)-\alpha(u)\right)\right)^{\ell} \leq C_{\ell} x^{\frac{\ell}{2}} \max _{1 \leq i \leq x} \mathbb{E}\left|Y_{i}(u)-\alpha(u)\right|^{\ell} \leq C_{\ell} x^{\frac{\ell}{2}},
$$

where $C_{\ell}$ is a universal constant. Then, we deduce from the previous equalities that there exist two constants $N_{1}$ and $N_{2}$ such that

$$
\delta_{x} Q_{u} V_{p} \leq \alpha(u)^{p} V_{p}(x)+M_{1} x^{p-1}+M_{2} .
$$

To check the drift condition in $\mathbf{F 1}$ for $m=1$, one can choose $\gamma>0$ such that $\lambda=\max _{u \in[0,1]} \alpha(u)^{p}+\gamma<1$ and $b=M_{2}+\left(\frac{\gamma}{M_{1}}\right)^{p-1}$. In this case, the minoration condition is satisfied on each finite set $C$ with $v=\delta_{0}$ because

$$
Q_{u}(x, 0) \geq\left(1-\max _{u \in[0,1]} \alpha(u)\right)^{x} \exp \left(-\max _{u \in[0,1]} \lambda(u)\right)
$$

and $\eta=\min _{u \in[0,1]} \min _{x \in C} Q_{u}(x, 0)>0$. This shows that assumption $\mathbf{F 1}$ is satisfied by taking $R$ large enough. Finally, we show A3. Let $u, v \in[0,1]$. Denoting $\bar{\lambda}=\max _{u \in[0,1]} \lambda(u)$ and by $\mu_{u}$ the Poisson distribution of parameter $\lambda(u)$, we have

$$
\max _{u \in[0,1]} \mu_{u} V_{p} \leq 1+\mathbb{E} N_{\bar{\lambda}}^{p}, \quad\left\|\mu_{u}-\mu_{v}\right\|_{V_{p}} \leq \sum_{k \geq 0} \frac{V_{p}(k)}{k !}\left(k \bar{\lambda}^{k-1}+\bar{\lambda}^{k}\right) \cdot|\lambda(u)-\lambda(v)|,
$$

where $\left(N_{t}\right)_{t \geq 0}$ is Poisson process of intensity 1. Moreover, if $v_{u}$ denotes the Bernoulli distribution of parameter $\alpha(u)$, we have $\left\|v_{u}-v_{v}\right\|_{V_{p}} \leq 3|\alpha(u)-\alpha(v)|$. From Lemma 6, we easily deduce that $\mathbf{F} 2$ holds for $\widetilde{V}=C V_{p+1}$ where $C$ is a positive real number. Note that we have $\sup _{u \in[0,1]} \pi_{u} \widetilde{V}<\infty$ because $\widetilde{V}$ also satisfies the drift and minoration condition.

Let us now give an estimator for parameter $(\alpha(u), \lambda(u))$. A natural estimate is obtained by localized least squares. Setting $a(u)=(\alpha(u), \lambda(u))^{\prime}$ and $\mathcal{Y}_{n, i}=\left(1, X_{n, i-1}\right)^{\prime}$. Then we define

$$
\hat{a}(u)=\arg \min _{\alpha} \sum_{i=2}^{n} e_{i}(u)\left(X_{n, i}-\mathcal{Y}_{n, i}^{\prime} \alpha\right)^{2}=\left(\sum_{i=2}^{n} e_{i}(u) \boldsymbol{y}_{n, i} \mathcal{Y}_{n, i}^{\prime}\right)^{-1} \sum_{i=2}^{n} e_{i}(u) X_{n, i} \mathcal{Y}_{n, i},
$$


where the weights $e_{i}(u)$ were defined in Subsection 2.3. Using our results and assuming that $b \rightarrow 0$ and $n b \rightarrow \infty$, we get

$$
\sum_{i=2}^{n} e_{i}(u) \mathbb{E} \mathcal{Y}_{n, i} \mathcal{Y}_{n, i}^{\prime}=\mathbb{E} \boldsymbol{y}_{i}(u) \mathcal{Y}_{i}(u)^{\prime}+O\left(b^{\kappa}+\frac{1}{n^{\kappa}}\right)=O\left(b^{\kappa}\right)
$$

In the same way, we have

$$
\sum_{i=2}^{n} e_{i}(u) \mathbb{E} X_{n, i} \mathcal{y}_{n, i}=\mathbb{E} X_{i}(u) \mathcal{y}_{i}(u)+O\left(b^{\kappa}+\frac{1}{n^{\kappa}}\right)=O\left(b^{\kappa}\right) .
$$

Moreover, using our covariance inequality (see the notes after Proposition 7), we get

$$
\operatorname{Var}\left(\sum_{i=2}^{n} e_{i}(u) \boldsymbol{y}_{n, i} \mathcal{Y}_{n, i}^{\prime}\right)=O\left((n b)^{-1}\right)
$$

Moreover using the decomposition $X_{n, i}=\mathcal{Y}_{n, i}^{\prime} a(i / n)+X_{n, i}-\mathbb{E}\left(X_{n, i} \mid \mathcal{F}_{n, i-1}\right)$ where $\mathcal{F}_{n, i}=\sigma\left(X_{n, j}: j \leq i\right)$ and the fact that for all $p \geq 1$, $\sup _{n \in \mathbb{Z}^{+}, k \leq n} \mathbb{E}\left|X_{n, k}\right|^{p}<\infty$, we also obtain

$$
\operatorname{Var}\left(\sum_{i=2}^{n} e_{i}(u) \mathcal{Y}_{n, i} X_{n, i}\right)=O\left((n b)^{-1}\right) .
$$

Collecting all the previous properties, we get $\hat{a}(u)=a(u)+O_{\mathbb{P}}\left(b^{\kappa}+\frac{1}{\sqrt{n b}}\right)$. Asymptotic normality or uniform control of $\hat{a}(u)-a(u)$ can also be obtained using adapted results for strong mixing sequences.

\section{Auxiliary results}

\subsection{Auxiliary result for Section 2}

Proposition 8. Let $\left(H_{i}^{(n)}\right)_{1 \leq i \leq n, n>0}$ be a double array of real-valued random variables with finite variance and mean zero. Let $\left(\alpha_{n}(k)\right)_{k \geq 0}$ be the sequence of strong mixing coefficients of the sequence $\left(H_{i}^{(n)}\right)_{1 \leq i \leq n, n>0}$ and $\alpha_{(n)}^{-1}$ be the inverse function of the associated mixing rate function. Suppose that

$$
\lim \sup _{n \rightarrow \infty} \max _{1 \leq i \leq n} \frac{V_{n, i}}{V_{n, n}}<\infty
$$

where $V_{n, i}=\operatorname{Var}\left(\sum_{j=1}^{i} H_{j}^{(n)}\right)$. Let

$$
Q_{n, i}=\sup \left\{t \in \mathbb{R}_{+}: \mathbb{P}\left(\left|H_{i}^{(n)}\right|>t\right)>u\right\}
$$

Then $\sum_{i=1}^{n} H_{i}^{(n)}$ converges to the standard normal distribution if

$$
V_{n, n}^{-3 / 2} \sum_{i=1}^{n} \int_{0}^{1} \alpha_{(n)}^{-1}(x / 2) Q_{n, i}^{2}(x) \inf \left(\alpha_{(n)}^{-1}(x / 2) Q_{n, i}(x), \sqrt{V_{n, n}}\right) d x \rightarrow 0,
$$

as $n$ tends to $\infty$. 


\subsection{Auxiliary Lemmas for Section 3}

Lemma 1. Let $\mu \in \mathcal{P}_{p}(E)$ and $Q, R$ be two probability kernels from $(E, \mathcal{B}(E))$ to $(E, \mathcal{B}(E))$ such that

1. for all $x \in E$, the two probability measures $\delta_{x} Q$ and $\delta_{x} R$ are elements of $\mathcal{P}_{p}(E)$,

2. there exists $C>0$ such that for all $(x, y) \in E^{2}$,

$$
W_{p}\left(\delta_{x} Q, \delta_{y} Q\right) \leq C d(x, y), \quad W_{p}\left(\delta_{x} R, \delta_{y} R\right) \leq C d(x, y) .
$$

Then, if $\mu \in \mathcal{P}_{p}(E)$, the two probability measures $\mu Q, \mu R$ are also elements of $\mathcal{P}_{p}(E)$. Moreover, we have

$$
W_{p}^{p}(\mu Q, \mu R) \leq \int W_{p}^{p}\left(\delta_{x} Q, \delta_{x} R\right) d \mu(x),
$$

and if $v$ is another element of $\mathcal{P}_{p}(E)$, we have

$$
W_{p}(\mu Q, v Q) \leq C W_{p}(\mu, \nu) .
$$

Proof of Lemma 1. Using Lemma 3 for $f(x)=d\left(x, x_{0}\right)$, we have for a given $y \in E$,

$$
\begin{aligned}
\int d\left(x, x_{0}\right)^{p} Q(y, d x) & \leq\left[W_{p}\left(\delta_{y} Q, \delta_{x_{0}} Q\right)+\left(\int d\left(x, x_{0}\right)^{p} Q\left(x_{0}, d x\right)\right)^{1 / p}\right]^{p} \\
& \leq\left[C d\left(x_{0}, y\right)+\left(\int d\left(x, x_{0}\right)^{p} Q\left(x_{0}, d x\right)\right)^{1 / p}\right]^{p} .
\end{aligned}
$$

After integration with respect to $\mu$, it is easily seen that $\mu Q \in \mathcal{P}_{p}(E)$.

To show (15), one can use Kantorovitch duality (see Villani (2009), Theorem 5.10). Denoting by $C_{b}(E)$ the set of bounded continuous functions on $E$, we have

$$
\begin{aligned}
W_{p}^{p}(\mu Q, \mu R) & =\sup _{\phi(x)-\psi(y) \leq d(x, y)^{p},(\phi, \psi) \in C_{b}(E)}\left\{\int \phi(x) \mu Q(d x)-\int \psi(y) \mu R(d y)\right\} \\
& \leq \int\left[\sup _{\phi(x)-\psi(y) \leq d(x, y)^{p},(\phi, \psi) \in C_{b}(E)}\left\{\int \phi(x) Q(z, d x)-\int \psi(y) R(z, d y)\right\}\right] \mu(d z) \\
& \leq \int W_{p}^{p}\left(\delta_{z} Q, \delta_{z} R\right) \mu(d z) .
\end{aligned}
$$

Finally, we show (16). Let $\phi, \psi$ be two elements of $C_{b}(E)$ such that $\phi(x)-\psi(y) \leq d(x, y)^{p}$ and $\gamma$ an optimal coupling for $(\mu, v)$. Then, for $u, v \in E$, we have

$$
\int \phi(x) Q(u, d x)-\int \psi(y) Q(v, d y) \leq W_{p}^{p}\left(\delta_{u} Q, \delta_{v} Q\right) \leq C^{p} d(u, v)^{p} .
$$

Moreover,

$$
\int \phi(x) \mu Q(d x)-\int \psi(y) v Q(d y)=\int \gamma(d u, d v)\left[\int \phi(x) Q(u, d x)-\int \psi(y) Q(v, d y)\right] .
$$

Then (16) easily follows from Kantorovitch duality. $\square$ 
Lemma 2. Let $j \geq 1$ be an integer. Assume that $Q_{1}, \ldots, Q_{j}$ and $R_{1}, \ldots, R_{j}$ are Markov kernels such that for all $x \in E$ and $1 \leq i \leq j, \delta_{x} Q_{i}$ and $\delta_{x} R_{i}$ are elements of $\mathcal{P}_{p}(E)$ satisfying

$$
W_{p}\left(\delta_{x} Q_{i}, \delta_{y} Q_{i}\right) \leq L_{i} d(x, y), \quad W_{p}\left(\delta_{x} R_{i}, \delta_{y} R_{i}\right) \leq L_{i} d(x, y),
$$

for all $(x, y) \in E^{2}$. Then, for all $x \in E$, we have

$$
W_{p}\left(\delta_{x} Q_{1} \cdots Q_{j}, \delta_{x} R_{1} \cdots R_{j}\right) \leq \sum_{s=0}^{j-1} L_{j} \cdots L_{j-s+1} D_{j-s}
$$

where $D_{i}^{p}=\int W_{p}^{p}\left(\delta_{y} Q_{i}, \delta_{y} R_{i}\right) \delta_{x} R_{1} \cdots R_{i-1}(d y)$.

Proof of Lemma 2 Using the inequality

$$
W_{p}\left(\delta_{x} Q_{1} \cdots Q_{j}, \delta_{x} R_{1} \cdots R_{j}\right) \leq \sum_{s=0}^{j-1} W_{p}\left(\delta_{x} R_{1} \cdots R_{j-s-1} Q_{j-s} \cdots Q_{j}, \delta_{x} R_{1} \cdots R_{j-s} Q_{j-s+1} \cdots Q_{j}\right),
$$

the result follows using Lemma 1.

Lemma 3. If $f: E \rightarrow \mathbb{R}$ is a Lipschitz function, then for all measures $\mu, v \in \mathcal{P}_{p}(E)$, we have

$$
\left|\left(\int f^{p} d \mu\right)^{1 / p}-\left(\int f^{p} d v\right)^{1 / p}\right| \leq \delta(f) W_{p}(\mu, v),
$$

where $\delta(f)$ denotes the Lipschitz constant of $f$ :

$$
\delta(f)=\sup _{x \neq y} \frac{|f(x)-f(y)|}{d(x, y)} .
$$

Proof of Lemma 3 If $\gamma$ denotes an optimal coupling for $(\mu, v)$, we get from the triangular inequality,

$$
\begin{aligned}
& \left|\left(\int f^{p} d \mu\right)^{1 / p}-\left(\int f^{p} d \nu\right)^{1 / p}\right| \\
= & \left|\left(\int f^{p}(x) d \gamma(x, y)\right)^{1 / p}-\left(\int f^{p}(y) d \gamma(x, y)\right)^{1 / p}\right| \\
\leq & \left(\int|f(x)-f(y)|^{p} d \gamma(x, y)\right)^{1 / p} \\
\leq & \delta(f)\left(\int d(x, y)^{p} d \gamma(x, y)\right)^{1 / p} .
\end{aligned}
$$

which leads to the result of the lemma.

Lemma 4. Let $X$ and $Y$ two random variables taking values in $(E, d)$ and such that $\mathbb{P}_{X}, \mathbb{P}_{Y} \in \mathcal{P}_{d}(E)$. On $E \times E$, we define the metric

$$
\widetilde{d}\left(\left(x_{1}, x_{2}\right),\left(y_{1}, y_{2}\right)\right)=\left(d\left(x_{1}, y_{1}\right)^{p}+d\left(x_{2}, y_{2}\right)^{p}\right)^{1 / p} .
$$

Then we have

$$
W_{p}\left(\mathbb{P}_{X, Y}, \mathbb{P}_{Y, Y}\right) \geq 2^{-\frac{p-1}{p}} \mathbb{E}^{1 / p}\left(d(X, Y)^{p}\right) .
$$


Proof of Lemma 4 Consider the Lipschitz function $f: E \times E \rightarrow \mathbb{R}$ defined by $f\left(x_{1}, x_{2}\right)=d\left(x_{1}, x_{2}\right)$. Using the triangular inequality and convexity, we have $\delta(f) \leq 2^{\frac{p-1}{p}}$. Then the result is a consequence of Lemma 3. $\square$

\subsection{Auxiliary Lemmas for Section 4}

The following result is an adaptation of Lemma 6.29 given in Douk et al. (2014). The proof is omitted because the arguments are exactly the same. See also Hairer and Mattingly (2011) for the original proof of this result. Note however, that we use the condition $R>\frac{2 b}{1-\lambda}$ instead of $R>\frac{2 b}{(1-\lambda)^{2}}$ because coefficients $(\lambda, b)$ are obtained for $m$ iterations of the kernel (in Douk et al. (2014), these coefficients are that for the case $m=1)$. For a Markov kernel $R$ on $(E, \mathcal{B}(E))$, we define its Dobrushin's contraction coefficient by

$$
\Delta_{V}(R)=\sup \left\{\frac{\|\mu R-v R\|_{V}}{\|\mu-v\|_{V}}: \quad \mu, v \in \mathcal{P}(E), \quad \mu V<\infty, v V<\infty\right\} .
$$

Lemma 5. Under assumption $\mathbf{F 1}$, there exists $(\gamma, \delta) \in(0,1)^{2}$, only depending on $\lambda, \eta, b$, such that for all $\left(u, u_{1}, \ldots, u_{m}\right) \in[0,1]^{m+1}$ such that $\left|u_{i}-u\right| \leq \epsilon, 1 \leq i \leq m$, we have

$$
\Delta_{V_{\delta}}\left(Q_{u_{1}} \cdots Q_{u_{m}}\right) \leq \gamma \text {, where } V_{\delta}=1-\delta+\delta V .
$$

Lemma 6. Let $X_{1}, X_{2}, \ldots, X_{n}, Y_{1}, Y_{2}, \ldots, Y_{n}$ be independent random variables such that $A_{n}=\max _{1 \leq i \leq n} \mathbb{E} V\left(X_{i}\right) \vee$ $\mathbb{E} V\left(Y_{i}\right)<\infty$ for $1 \leq i \leq n$, with $V(x)=1+|x|^{p}$ and $p \geq 1$. Then we have

$$
\sup _{|f| \leq V}\left|\mathbb{E} f\left(X_{1}+\cdots+X_{n}\right)-\mathbb{E} f\left(Y_{1}+\cdots+Y_{n}\right)\right| \leq 2^{p+1} n^{p+1} \cdot A_{n} \cdot \max _{1 \leq i \leq n}\left\|\mathbb{P}_{X_{i}}-\mathbb{P}_{Y_{i}}\right\|_{V} .
$$

Proof of Lemma 6. Note first that if $|f(x)| \leq V(x)$ for all $x \in E$, then $|f(x+y)| \leq 2^{p} V(x) V(y)$. This leads to

$$
\begin{aligned}
& \left|\mathbb{E} f\left(X_{1}+\cdots+X_{n}\right)-\mathbb{E} f\left(Y_{1}+\cdots+Y_{n}\right)\right| \\
\leq & \sum_{j=1}^{n}\left|\mathbb{E} f\left(X_{1}+\cdots+X_{j-1}+X_{j}+Y_{j+1}+\cdots+Y_{n}\right)-f\left(X_{1}+\cdots+X_{j-1}+Y_{j}+Y_{j+1}+\cdots+Y_{n}\right)\right| \\
\leq & 2^{p} \sum_{j=1}^{n}\left\|\mathbb{P}_{X_{j}}-\mathbb{P}_{Y_{j}}\right\|_{V} \cdot \mathbb{E} V\left(X_{1}+\cdots+X_{j-1}+Y_{j+1}+\cdots+Y_{n}\right) \\
\leq & 2^{p}(n-1)^{p} \sum_{j=1}^{n}\left\|\mathbb{P}_{X_{j}}-\mathbb{P}_{Y_{j}}\right\|_{V} A_{n} \\
\leq & 2^{p} n^{p+1} A_{n} \max _{1 \leq i \leq n}\left\|\mathbb{P}_{X_{i}}-\mathbb{P}_{Y_{i}}\right\|_{V} \cdot \square
\end{aligned}
$$

\section{References}

M. Al Osh and A. Alzaid. Firs-order integer-valued autoregressive process. J. Time Series Anal., 8:261-275, 1987.

R. Dahlhaus. Fitting time series models to nonstationary processes. Ann. Statist., 25:1-37, 1997. 
R. Dahlhaus and S. Subba Rao. Statistical inference for time-varying arch processes. Ann. Statist., 34: 1075-1114, 2006.

J. Dedecker and C. Prieur. Coupling for $\tau$-dependent sequences and applications. Journal of Theoretical Probability, 17:861-885, 2004.

R.L. Dobrushin. Central limit theorems for nonstationary markov chains. Th. Prob. Appl., 1:329-383, 1956.

R.L. Dobrushin. Prescribing a system of random variables by conditional distributions. Th. Prob. Appl., 15: 458-486, 1970.

R. Douc, E. Moulines, and J.S. Rosenthal. Quantitative bounds on convergence of time-inhomogeneous markov chains. Ann. Appl. Probab., 14(4):1643-1665, 2004.

R. Douk, E. Moulines, and D. Stoffer. Nonlinear Time Series. Chapman and Hall, 2014.

P. Doukhan. Mixing. Properties and Examples. Springer-Verlag, 1994.

C. Francq and J-M. Zakoïan. GARCH models: structure, statistical inference and financial applications. Wiley, 2010.

P. Fryzlewicz, T. Sapatinas, and S. Subba Rao. Normalized least-squares estimation in time-varying arch models. Ann. Statist., 36:742-786, 2008.

M. Hairer and J.C. Mattingly. Yet another look at harris' ergodic theorem for markov chains. In Seminar on Stochastic Analysis, Random Fields and Applications IV, volume 63, pages 109-117. Birkhäuser,/Springer Basel AG, Basel, 2011.

D. Jin-Guan and L. Yuan. The integer-valued autoregressive (inar(p)) model. J. Time Series Anal., 12: 129-142, 1991.

S. Meyn and R.L. Tweedie. Markov Chains and Stochastic Stability 2nd. Cambridge University Press New York, 2009.

E. Rio. About the lindeberg method for strongly mixing sequences. ESAIM, Probability and Statistics, 1: 35-61, 1995.

E. Rio. Théorie asymptotique des processus aléatoires faiblement dépendants. Springer, 1999.

E. Rio. Inequalities and limit theorems for weakly dependent sequences. https://cel . archives-ouvertes/cel-00867106, 2013.

L. Saloff-Coste and J. Zúñiga. Convergence of some time-inhomogeneous markov chains via spectral techniques. Stochastic Process. Appl., 117:961-979, 2007.

L. Saloff-Coste and J. Zúñiga. Merging for inhomogeneous finite markov chains, part ii: Nash and logsobolev inequalities. Ann. Probab., 39:1161-1203, 2011.

S. Subba Rao. On some nonstationary, nonlinear random processes and their stationary approximations. Adv. in App. Probab., 38:1155-1172, 2006. 
L. Truquet. Parameter stability and semiparametric inference in time-varying arch models. Forthcoming in JRSSB, 2016.

C. Villani. Optimal Transport. Old and New. Springer, 2009.

M. Vogt. Nonparametric regression for locally stationary time series. Ann. Statist., 40:2601-2633, 2012.

G. Winkler. Image Analysis, Random Fields and Dynamic Monte Carlo Methods. Springer, 1995.

T. Zhang and W.B. Wu. Time-varying nonlinear regression models: nonparametric estimation and model selection. Ann. Statist., 43:741-768, 2015. 